\title{
A Reconciliation of Packed Column Permeability Data: Deconvoluting the Ergun Papers
}

\author{
Hubert M. Quinn \\ The Wrangler Group LLC, 40 Nottinghill Road, Brighton, MA 02135, USA \\ Correspondence should be addressed to Hubert M. Quinn; hubert@wranglergroup.com
}

Received 23 May 2014; Revised 18 July 2014; Accepted 21 July 2014; Published 22 September 2014

Academic Editor: Te-Hua Fang

Copyright ( 2014 Hubert M. Quinn. This is an open access article distributed under the Creative Commons Attribution License, which permits unrestricted use, distribution, and reproduction in any medium, provided the original work is properly cited.

\begin{abstract}
In his 1952 publication, Ergun made the following proclamation: "Data of the present investigation and those presented earlier have been treated accordingly, and the coefficients $k_{1}$ and $k_{2}$ have been determined by the method of least squares. The values obtained are $k_{1}=150$ and, $k_{2}=1.75$, representing 640 experiments." In this paper, we demonstrate that because his experimental methodology was flawed, the corrected values, which his experimental results would otherwise have established for these coefficients, are significantly higher. This is, in part, because Ergun's reporting of his measured data was ambiguous with respect to the embedded coefficients $k_{1}$ and $k_{2}$. In addition, this ambiguity made it difficult for any subsequent researcher to figure out the true meaning of his empirical results which, in turn, resulted in his choice of the values for these coefficients being accepted by default in the scientific community.
\end{abstract}

\section{Introduction}

The Ergun equation is unquestionably the most popular equation in use at this time to represent the relationship between pressure drop and resultant fluid flow in packed beds, when the fluid profile contains significant contribution from kinetic effects. In addition, most engineering departments at major universities and third level educational institutions throughout the world offer a teaching module which has as its focus the now famous "Ergun equation." The engineering literature, both chemical and otherwise, is replete with references to the equation and many of the studies reported therein have used it as a benchmark, against which to compare and contrast their empirical results. The equation was developed by Sabri Ergun in the late 1940s/early 1950s. It was derived, in part, from experimental measurements and, in part, from theoretical postulates. Ergun's initial paper was published in 1949 in collaboration with Orning. In two follow-up papers published in 1951 and 1952, Ergun reported his empirical results of enormous amounts of experimental runs aimed, in part, at calibrating the equation in terms of its two built-in coefficients. In this paper, we review these papers on this subject matter which we refer to collectively as the "Ergun papers."
The first empirical data on the relationship between fluid flow rate and pressure drop across a packed bed was reported by Darcy in 1856 [1]. The resultant Darcy law states that when the flow is streamline, the fluid flow rate through a packed bed is proportional to the pressure drop across the bed. The law has limitations, however, because it only applies to streamline flow and, in addition, tells us nothing about the factors which go into the constant of proportionality between fluid velocity and column pressure drop. Kozeny and Blake were the first to define in some greater detail the elements that define the constant of proportionality in Darcy flow $[2,3]$. The Kozeny-Blake equation, subsequently modified by Carman to accommodate irregularly shaped particles, has been used extensively to evaluate streamline flow but the coefficient therein has been the source of much controversy over the past fifty-plus years [4-9].

Scheidegger, in his textbook at page 89 , states: "It is obviously quite possible for two porous media of the same porosity to have entirely different permeabilities [10]. Thus, if a correlation function between the two quantities is sought after, it cannot be unique. Therefore, most empirical correlations contain some other factors, usually vaguely identified with alleged geometrical quantities. They are, however, nothing but undetermined factors used in order to make the data 
fit the desired equations. There are even a series of claims for "general" relationships, usually supposed to be true for "average" porous media, whatever that means." He continues: “Thus, Fancher, Lewis and Barnes $(1933,1934)$ and Manegold (1937) observed that air permeabilities are higher than liquid permeabilities in the same porous medium as calculated from Darcy's law. In fact, this is an indication that Darcy's law is not valid for gases."

In the textbook, Transport Phenomena, 1960 edition, the authors accept the value of 150 for the viscous coefficient in the Kozeny-Blake equation [11]. At page 199 of the book, which has become the gold standard at most chemical engineering schools, the authors state: "Analysis of a great deal of data has led to the value of 25/6, which we accept here." They go on to qualify its use as being valid for column porosities less than 0.5 and insert this ratio into the equation to show that it translates into the value of 150 which would correspond to the value of $k_{1}$ in the Ergun equation. Curiously, however, they provide no reference for the "great deal of data" so there is no way for us to determine from whence it came. In the second edition of the text, published in 2002, at page 190 the authors further state that "it has been found that replacing 16 by $100 / 3$ allows the tube bundle model to describe the packedcolumn data." They go on to show that this choice of ratio, though different from the one quoted in the previous edition of the book, results in the same value of 150 for the viscous coefficient and, yet again, no reference given as a foundation.

Happel and Brenner, in their textbook state: "For packed beds of uniform spheres the Carman-Kozeny equation within the range $\varepsilon=0.26$ to $\varepsilon=0.48$, gives excellent correlation with a Kozeny constant of $k=4.8$ [12]. A recent study by Anderson, including additional sources, indicates that for uniform spheres $4.2 \leq k \leq 6$. Anderson proposes a refinement in which $k$ is taken as a function of $\varepsilon$ instead of being assumed constant. A large volume of data on beds consisting of a variety of non-spherical particles indicate that $k \approx 5.0$, independent of shape and porosity from $\varepsilon=0.26$ to $\varepsilon=0.8$. As shown in Table 8-4.2, agreement of the Carman-Kozeny relationship with hydrodynamic theory based on the free surface cell model is excellent." It will be appreciated that a Kozeny constant of 5.0 corresponds to a value of $180(5 \times 36)$ for the coefficient in the Kozeny-Blake equation, as modified by Carman.

Stuart Winston Churchill, at page 524 of his textbook states: "Ergun combined the Carman-Kozeny equation but with a coefficient of 150 instead of 180" [13]. He goes on to show a plot of Ergun's data which he describes in the caption as "Comparison of experimental data for the pressure drop through packed beds of spheres, cylinders, sand, pulverized coke, and Raschig rings. . from Ergun."

In his handbook, Uwe Dieter Neue states: "As we know from experience, the backpressure of a column packed with small particles is larger than the backpressure of a column packed with large particles...The relationship is known as the Kozeny-Carman equation" [14]. This author goes on to identify the value of 185 as the coefficient in his version of the equation.

Dullien, in his textbook, at page 246 states: "MacDonald et al., have tested the Ergun equation using much more data than was ever used before by others [15]. The following relations were formed to give the best fit to all of the data." Dullien, who was also a coauthor on the MacDonald paper, then points out that, for smooth particles, values of 180 and 1.8 , for $k_{1}$ and $k_{2}$, are best, but for roughest particles the value of 1.8 should be replaced by the value of 4 .

Rhodes, in his textbook at page 85, shows the Ergun equation with the typical coefficients 150 and 1.75 but also includes the value of 180 for the viscous constant in what he describes as the Carman-Kozeny equation [16].

Guiochon et al., in their textbook state at page 152: "It has been claimed sometimes that spherical particles have a larger permeability than irregular-shaped ones, but definitive data are not available. The effect, as far as it exists, may in part result from uncertainties in the definition and measurement of the average size of irregular particles" [9].

Finally, Giddings, in his first textbook published in 1965, at page 209, displays in Table 5.3-1 arguably the most comprehensive data summary of packed column permeability in the published literature [7]. This data, when analyzed with respect to the form and function of the Kozeny-Blake equation, establishes a value of 267 for the viscous constant which corresponds to the value of $k_{1}$ in the Ergun equation [4]. In addition, at page 65 of his second textbook, published in 1991, the author displays what he refers to as the Kozeny-Carman equation with the value of 180 for the viscous coefficient. In a footnote below the equation, however, the author adds the parenthetic qualification "(This equation appears to work better for chromatographic materials if 180 is replaced by 270)" [8].

Ergun and Orning were the first to piece together an equation for flow in porous media which contained a viscous term and kinetic term and in 1952 Ergun announced specific values for the equation coefficients, $k_{1}$ and $k_{2}$. These values for the coefficients, however, have not been shown to be universally effective at correlating empirical values [17-19].

\section{The 1949 Paper}

We begin our discussion of the Ergun papers by going back to Ergun and Orning's original 1949 paper, not only because it was the genesis of the Ergun equation, but also because it planted the seeds of error which eventually led Ergun to come up with his mistaken values for the equation residual coefficients, $k_{1}$ and $k_{2}$, in 1952. Ergun and Orning begin with Kozeny's equation for viscous flow, which they say was proposed by Carman for use with liquids, and extended for use with gases by Lea and Nurse. The authors then move to a discussion of packed columns under high flow rate conditions in which the pressure drop appears to vary with some power of the velocity, the exponent ranging between 1 and 2. They note that Blake postulated that this fluid behavior was analogous to that in open tubes and, thus, he proposed an equation which was based on a friction factor meant to normalize for the kinetic effects in packed beds of granular material. This friction factor, in turn, was a function of the particle based Reynolds number.

The authors next address the collective effects of the viscous and kinetic contributions to overall pressure drop, noting that there is a smooth transition from viscous-dominated to kinetic-dominated effects. They argue, therefore, that there 
should be a continuous function relating pressure drop to flow rate. They conclude that "a general relationship may be developed using the Kozeny's assumption that the granular bed is equivalent to a group of parallel and equal-sized channels, such that the total internal surface and the free internal volume are equal to the total packing surface and the void volume, respectively, of the randomly packed bed." The authors now turn to Poiseuille's equation for flow through an open channel:

$$
\frac{d P}{d L}=\frac{32 \mu_{i} \eta}{d_{c}^{2}}
$$

where $d P / d L=$ pressure gradient across channel, $\mu_{i}=$ average interstitial fluid velocity, $d_{c}=$ diameter of capillary, and $\eta=$ fluid absolute viscosity.

Taking a term analogous to that introduced by Brillouin to represent the kinetic energy losses in a capillary, the authors add it to Poiseuille's equation:

$$
\frac{d P}{d L}=\frac{32 \mu_{i} \eta}{d_{c}^{2}}+\frac{\mu_{i}^{2} \rho_{f}}{2 d_{c}}
$$

where $\rho_{f}=$ fluid density.

At this point, Ergun and Orning postulate the number of channels per unit area and their size can be eliminated in favor of specific surface and fractional void volume and reexpress their equation as

$$
\frac{d P}{d L}=\frac{2\left(1-\varepsilon_{0}\right)^{2} S_{v}{ }^{2} \mu_{s} \eta}{\varepsilon_{0}{ }^{3}}+\frac{\left(1-\varepsilon_{0}\right) S_{v} \mu_{s}{ }^{2} \rho_{f}}{8 \varepsilon_{0}{ }^{3}},
$$

where $\varepsilon_{0}=$ column external porosity, $S_{v}=$ particle specific surface area, and $\mu_{s}=$ fluid superficial linear velocity.

The authors point out that although this equation is similar to others which relate pressure drop to polynomials of the flow rate, it differs to the extent that the coefficients, that is, 2 and $1 / 8$, have definite theoretical significance. Presumably, they conclude this because these values have their genesis in the physical relationships pertaining to cylindrical channels.

They further theorize that, for a packed bed, "the flow path is sinuous and the streamlines frequently converge and diverge. The kinetic losses, which occur only once for the capillary, occur with a frequency that is statistically related to the number of particles per unit length. For these reasons, a correction factor must be applied to each of the energy loss terms. These factors may be designated as $\alpha$ and $\beta$." Accordingly, they reexpress (3) as follows:

$$
\frac{d P}{d L}=\frac{2 \alpha\left(1-\varepsilon_{0}\right)^{2} S_{v}{ }^{2} \mu_{s} \eta}{\varepsilon_{0}{ }^{3}}+\frac{\beta\left(1-\varepsilon_{0}\right) S_{v} \mu_{s}{ }^{2} \rho_{f}}{8 \varepsilon_{0}{ }^{3}} .
$$

They point out that $\alpha$ and $\beta$ may be determined experimentally. They claim that experiments with randomly packed materials of known specific surface area have led to the adoption of a value of 5 for $2 \alpha$, which is close to the theoretical value of $(\pi / 2)^{2}$, or 2.47 , as proposed by Hitchcock [20].

The authors then assert that integration of (4), assuming isothermal expansion of an ideal gas, leads to the equation

$$
\frac{\Delta P}{L \mu_{s}{ }^{m}}=\frac{2 \alpha\left(1-\varepsilon_{0}\right)^{2} S_{v}{ }^{2} \eta}{\varepsilon_{0}{ }^{3}}+\frac{\beta\left(1-\varepsilon_{0}\right) S_{v}\left(q_{m} / A\right)}{8 \varepsilon_{0}{ }^{3}},
$$

where $\mu_{s}^{m}=$ average superficial linear velocity of fluid (gas) at the mean pressure, $q_{m}=$ mass flow rate, and $A=$ column cross-sectional area.

The authors state that Leva and Grummer reported that, in packed tubes, when the viscous forces are predominant, the pressure drop is proportional to $\left[\left(1-\varepsilon_{0}\right)^{2} / \varepsilon_{0}{ }^{3}\right]$, whereas, at higher flow rates, the corresponding dependence on the void fraction is $\left[\left(1-\varepsilon_{0}\right) / \varepsilon_{0}{ }^{3}\right]$, which is just what would be expected from (5) [21]. They then point out that a plot of $\Delta P / L \mu^{m}$ versus $q_{m} / A$ would give a straight line, the intercept and slope leading to the values of $\alpha$ and $\beta$.

In Figure 1 of their 1949 paper, they display their own measurements in the form of what they say are "Typical plots of data obtained to test this dependence of pressure drop on gas flow rate." However, the ordinate of the plot contains the velocity designated as $U_{m}$ (not $u_{m}$ ). This symbol appears nowhere else in the paper and is conspicuously absent from the glossary of terms at the end of the paper. We interpret this to mean, however, that the authors intentionally use interchangeably the superficial velocity at the mean pressure and at operating conditions of standard temperature and pressure STP. (This is also the same protocol used by Ergun in his 1952 paper. See his discussion in that publication of his equation (10) relative to his Figure 4.) Additionally, the coordinates of the plot have units of measure corresponding to a plot of $\Delta P / L \mu$ on the ordinate against $q_{m} / A$ on the abscissa. The plot contains 5 different sets of measurements which exhibit straight lines with varying slope and intercept values. In their Figures 2 and 3, they show two additional plots in which they have taken data from the work of Burke and Plummer and Oman and Watson, respectively, which also give straight lines, thus allegedly validating (7) [22, 23]. (The ordinates of these plots have the velocity also designated as $U_{m}$.) Additionally, in their Table 1 , they provide values for $\alpha$ and $\beta$ which range from 1.7 to 3.0 and from 2.4 to 4.5 , respectively, which they derive from numerous data sets, including their own.

The authors state that, using the values of $\alpha$ and $\beta$ from Table 1 and considering their (8), one can discern the relative importance of the viscosity and the kinetic terms at different Reynolds numbers. Specifically, they point out that, for Reynolds numbers around 60 and a column porosity of 0.35 , the two have nearly equal effects upon pressure drop. However, for a Reynolds number of 0.1 , viscosity accounts for $99.8 \%$ of the pressure drop, while, for a Reynolds number of 3000 , kinetic effects account for $97 \%$ of the pressure drop. They go on to point out that because packed columns are generally operated in the range from Reynolds numbers of 1 to 10,000 , both terms are essential in calculations of pressure drops.

They conclude their discussion on fixed beds with the statement that when using geometrically arranged packing, as opposed to random packing, (5) is valid except that the coefficients may vary markedly for different arrangements, even at the same fractional void volumes.

2.1. The Theoretical Parameters $\alpha$ and $\beta$. In describing the role of $\alpha$ and $\beta$, the authors say that "For a packed bed the 
flow path is sinuous and the streamlines frequently converge and diverge. The kinetic losses, which occur only once for the capillary, occur with a frequency that is statistically related to the number of particles per unit length. For these reasons, a correction factor must be applied to each term. These factors may be designated as $\alpha$ and $\beta$."

This postulate raises more questions than answers. Assuming, for example, that the statement concerning the kinetic losses is correct, why does this necessitate a correction factor for the viscous term? After all, the adoption of Kozeny's postulate for streamline flow regarding the equivalency of the granular bed to open channels supposedly captures all the flow considerations in the viscous term. Another obvious question relates to the rationale underlying the assertion that the kinetic losses "occur only once for the capillary." Finally, since the authors abandoned any effort to relate pressure drop to the array of individual particles in a packed bed in favor of a theoretical model of equivalent channels, the concept of kinetic losses being related to "the number of particles per unit length" would appear to be out of place in this newly adopted "channel" model.

The authors attempt to justify their values for $\alpha$ and $\beta$ in their (9), which includes an expression relating an entity, $f$, which they call "the friction factor" to both $\alpha$ and $\beta$. We repeat that equation here:

$$
f=\beta\left(1+\frac{96 \alpha}{\beta R_{\mathrm{em}}^{\mathrm{stp}}}\right),
$$

where $R_{\mathrm{em}}{ }^{\text {stp }}=$ the modified Reynolds number at STP.

(Note: the author's (9) has a misprint. The $\alpha$ which appears outside the bracket on the right hand side of the equation should be $\beta$.)

Presumably, this is supposed to demonstrate that their values for $\alpha$ and $\beta$ can be derived from empirical data but it is not superficially obvious where the value of 96 comes from or what it represents. Characteristically, the authors do not explain how they arrived at their (9) from their starting equation (7).

It follows, however, that their (9) cannot by itself lead to independent values of $\alpha$ and $\beta$ since there are two unknowns and only one equation. In fact, their lone friction factor identified in their (9) is based on commingling of values associated with both the correlation coefficients and the independent variables in both the viscous and the kinetic terms of their equation. This commingling of the terms which make up the separate and distinct contributions of viscous and kinetic elements renders the resultant correlation coefficient (96) meaningless. In the end, therefore, both of the values of $\alpha$ and $\beta$ must be derived from the linear plot in Figure 1 and cannot be derived from the lone friction factor which serves only to underscore the interdependence of the one upon the other. The values of $\alpha$ and $\beta$, therefore, are without specific meaning since they represent some unknown mixture of both viscous and kinetic contributions.

2.2. The Mean Pressure Postulate. In our discussions to this point the relationships between $\mu_{s}, \mu^{m}$, and $q_{m}$ are implicit. We use the term $\mu_{s}$ to represent the superficial velocity of the fluid when the fluid is a liquid and thus not compressible, whereas the symbol $\mu^{m}$ is used when the fluid is compressible as in the case of gases. These relationships are

$$
\begin{aligned}
& \mu^{m}=\frac{q_{m}}{A \rho_{f m}}, \\
& \mu_{s}=\frac{q_{m}}{A \rho_{f}},
\end{aligned}
$$

where $\rho_{f m}=$ the density of the fluid at the mean pressure.

We may now express the ratio of $\mu^{m}$ and $\mu_{s}$. Thus,

$$
\frac{\mu^{m}}{\mu_{s}}=\frac{q_{m} /\left(A \rho_{f m}\right)}{q_{m} /\left(A \rho_{f}\right)} .
$$

It follows that

$$
\begin{gathered}
\mu^{m}=\frac{\rho_{f} \mu_{s}}{\rho_{f m}}, \\
\mu_{s}=\frac{\rho_{f m} \mu^{m}}{\rho_{f}} .
\end{gathered}
$$

The mass flow rate $q_{m}$ is related to the volumetric flow rate $q$ through the density of the fluid. Thus, we may write the following:

$$
\mu_{s}=\frac{q_{m}}{A \rho_{f}}=\frac{q}{A}
$$

It follows that

$$
q_{m}=q \rho_{f}
$$

where $q=$ the volumetric flow rate of the fluid.

We note from this relationship that there are an infinite number of combinations of $q$ and $\rho_{f}$ which will produce an equivalent value of $q_{m}$.

The author's theoretical postulate concerning the "mean pressure" is the equivalent of measuring the pressure drop at the inlet and outlet of the column and equating the average of the two values to the $\Delta P$ in (5). The $\Delta P$ in (5), however, is simply the difference between the pressure at the inlet and outlet of the column, not the average of the two. In addition, it represents the theoretical differential pressure drop since all other parameters in the equation are separately identified in the stated equation format. Let us, therefore, insert the theoretical boundary condition pertaining to the measured pressure drop, which is contained in their postulate of "mean pressure," on the left hand side of (5). We accomplish this by substituting $\Delta P / 2$ into the equation for $\Delta p$. Thus, this gives

$$
\frac{\Delta P}{2 L \mu^{m}}=\frac{k_{1}\left(1-\varepsilon_{0}\right)^{2} \eta}{\varepsilon_{0}^{3} d_{p}^{2}}+\frac{k_{2}\left(1-\varepsilon_{0}\right)\left(q_{m} / A\right)}{\varepsilon_{0}^{3} d_{p}},
$$

where $S_{v}=6 / d_{p}, k_{1}=72 \alpha$, and $k_{2}=3 / 4 \beta$.

We digress here to point out a potential problem in interpreting (12). Note that the left hand side of the equation 
has the velocity specified as that at the mean pressure, $\mu^{m}$. This is a very specific velocity; it is separately identified with its own symbol and discrete value but exists only in the theoretical context of Ergun and Orning's equation. The right hand side of (12), on the other hand, has the velocity embedded in the parameter $q_{m}$, as we pointed out above. In addition, since a given absolute value of $q_{m}$ can have an infinite number of combinations of fluid velocity and fluid density, (12), as stated, is ambiguous with respect to velocity on the right hand side. Restating (12) in unambiguous terms requires a substitution for the mass flow rate, $q_{m}$, to expose the embedded values of the velocity and density, as follows.

Substituting for $q_{m} / A=\mu^{m} \rho_{f m}$ gives

$$
\frac{\Delta P}{2 L \mu^{m}}=\frac{k_{1}\left(1-\varepsilon_{0}\right)^{2} \eta}{\varepsilon_{0}{ }^{3} d_{p}{ }^{2}}+\frac{k_{2}\left(1-\varepsilon_{0}\right) \mu^{m} \rho_{f m}}{\varepsilon_{0}{ }^{3} d_{p}} .
$$

Substituting for $\mu^{m}=\mu_{s}\left[\rho_{f} / \rho_{f m}\right]$ gives

$$
\frac{\Delta P \rho_{f m}}{2 L \mu_{s} \rho_{f}}=\frac{k_{1}\left(1-\varepsilon_{0}\right)^{2} \eta}{\varepsilon_{0}^{3} d_{p}^{2}}+\frac{k_{2}\left(1-\varepsilon_{0}\right) \mu_{s} \rho_{f}}{\varepsilon_{0}^{3} d_{p}} .
$$

Isolating the theoretical "mean pressure" gradient on the left hand side, that is, multiplying across by the quantity $\mu_{s} \rho_{f}$, gives

$$
\frac{\Delta P \rho_{f m}}{2 L}=\frac{k_{1}\left(1-\varepsilon_{0}\right)^{2} \mu_{s} \rho_{f} \eta}{\varepsilon_{0}{ }^{3} d_{p}{ }^{2}}+\frac{k_{2}\left(1-\varepsilon_{0}\right) \mu_{s}^{2} \rho_{f}^{2}}{\varepsilon_{0}{ }^{3} d_{p}} .
$$

The expression, $\Delta P \rho_{f m} / 2 L$, on the left hand side of (15), is the theoretical equivalent to the measured "mean pressure" gradient. In the theoretical version both the mean pressure gradient, $\Delta P / 2 L$, and the fluid density, $\rho_{f m}$, are separately identified. In the measured value, however, the effect of the density is embedded in the measured value of the pressure gradient and is not separately identified and, of course, there is no 2. We note that this theoretical equation now establishes a relationship between measured pressure gradient at the mean pressure, on the left hand side of (15), and superficial velocity at STP, which involves only quantities which we can measure outside the column, on the right hand side of the equation. Thus, we can establish a correlation between measured mean pressure and superficial velocity at STP. Note, however, that this procedure embeds the density at STP, $\rho_{f}$, into the viscous term as well as embedding the square of the density at STP, $\rho_{f}^{2}$, into the kinetic term.

Normalizing for $2 / \rho_{f}$ in (15) gives

$$
\frac{\Delta P \rho_{f m}}{L \rho_{f}}=\frac{2 k_{1}\left(1-\varepsilon_{0}\right)^{2} \mu_{s} \eta}{\varepsilon_{0}{ }^{3} d_{p}^{2}}+\frac{2 k_{2}\left(1-\varepsilon_{0}\right) \mu_{s}^{2} \rho_{f}}{\varepsilon_{0}^{3} d_{p}} .
$$

We can see then that this procedure requires us to adjust the theoretical pressure gradient at the mean pressure, that is, the left hand side of the equation, that is, $\Delta P / L$, by the ratio of the density at the mean pressure to the density at STP $\left(\rho_{f m} / \rho_{f}\right)$. This is necessitated because our reference superficial velocity on the right hand side of (16) is reflective of the fluid density at STP, $\rho_{f}$, when the flow rates were recorded. Therefore, we must "back out" the bias in the measured pressure gradient on the left hand side of the equation by an amount equal to the effect of the embedded ratio of the densities at the mean pressure and STP, in order to maintain parity between the two scenarios.

Equation (16) now represents the properly adjusted theoretical pressure/flow equation, based upon the author's mean pressure postulate, which allows us to correlate the measured pressure gradient of a compressible fluid to that of the volumetric velocity measured at STP at the outlet of the column. In fact, this equation format is valid whatever the reference density that one chooses to measure the volumetric flow rate at assuming of course that the density at STP, $\rho_{f}$, is replaced by the appropriate reference density $\rho_{f}{ }^{\text {ref }}$. Note, however, that this postulate has embedded a value of 2 into the right hand side of the equation. This means that author's postulate concerning the mean pressure has an embedded assumption that the ratio of the actual superficial velocity inside the column to that at STP is 2. We can show this mathematically as follows:

$$
\text { Let } \frac{\rho_{f m}}{\rho_{f}}=2 \text {. }
$$

Substituting for $\rho_{f m} / \rho_{f}$ in (16) gives

$$
\frac{2 \Delta P}{L}=\frac{2 k_{1}\left(1-\varepsilon_{0}\right)^{2} \mu_{s} \eta}{\varepsilon_{0}^{3} d_{p}^{2}}+\frac{2 k_{2}\left(1-\varepsilon_{0}\right) \mu_{s}^{2} \rho_{f}}{\varepsilon_{0}^{3} d_{p}} .
$$

Dividing across by 2 gives

$$
\frac{\Delta P}{L}=\frac{k_{1}\left(1-\varepsilon_{0}\right)^{2} \mu_{s} \eta}{\varepsilon_{0}^{3} d_{p}^{2}}+\frac{k_{2}\left(1-\varepsilon_{0}\right) \mu_{s}^{2} \rho_{f}}{\varepsilon_{0}^{3} d_{p}} .
$$

We can see then that (19) is a valid relationship between pressure gradient and the other independent variables whenever all variables are taken within the same frame of reference. In other words, when applied to a packed granular bed all parameters must be measured either inside the column or outside the column. Of course the measurement of pressure gradient outside the column does not make much sense and, therefore, this equation is only valid when the fluid is incompressible as in the case of a liquid where the density parameter is a constant value regardless of whether it is measured inside or outside the column. Accordingly, when using liquids it is customary to measure the pressure inside the column and the flow rate outside the column.

Thus, we restate (19) in order to capture the effect of fluid compressibility in the measured pressure drop as follows:

$$
\frac{\Delta P_{m}}{L}=\frac{k_{1}\left(1-\varepsilon_{0}\right)^{2} \mu_{s} \eta}{\varepsilon_{0}^{3} d_{p}^{2}}+\frac{k_{2}\left(1-\varepsilon_{0}\right) \mu_{s}^{2} \rho_{f}}{\varepsilon_{0}^{3} d_{p}} .
$$

Equation (20) is the correct equation format to be used when a compressible gas is used to generate pressure inside a column and the mass flow rate is measured outside the column. This format properly identifies the measured pressure drop 
and also the fact that the effect of fluid compressibility is embedded within the measured pressure drop. Therefore, (19) and (20) are identical representations of the same relationship with the proviso that the former represents the theoretical pressure drop (nothing unaccounted for embedded) but the latter represents the measured pressure drop which has embedded quantities which are not accounted for on the right hand side of the equation. Accordingly, (20) is not overtly balanced.

\section{The 1951 Paper}

Ergun, this time as the sole author in 1951, begins by noting that an exact knowledge of particle solid density is important for determining the void volume fraction (external porosity) in a column comprised of porous particles. This is because, without a direct independent method to measure the void volume fraction of a packed column, the usual practice is to measure the mass of particles in the packed column which enables the calculation of the column packing density. Using the column packing density, in turn, enables the calculation of the void volume fraction if the particle solid density is known. Unlike nonporous solids, however, the particle density of porous solids is not equivalent to the particle solid density and is a function of the particle size. The particle density of porous particles always increases as the particle size is decreased and becomes equivalent to the particle solid density when the particles are reduced to being nonporous. This results from the fact that as a given particle size of porous solids is further broken down to smaller particle sizes, some of the pores in the material are eliminated. Thus, in order to determine the particle solid density one must reduce the particle size to that of the smallest size of the pores within the particle. It follows that the particles may then be considered nonporous. This same principle also applies to the determination of the specific surface area of any porous particles.

Ergun continues that, because of the lack of a successful method to determine directly the particle solid density of porous particles, it has been generally the practice to assign an apparent particle density and use empirical adjustment factors such as shape and surface factors to correct for this lack of knowledge of the particle solid density. He says that a method to circumvent this problem is to consider a porous particle as a nonporous particle surrounded by an impervious volume envelope. In this scenario, therefore, the particle solid density is then the mass of the particles divided by the impervious volume envelope. The mass of the particles is easily determined but the impervious volume envelope is not. If, however, gas is made to flow through a packed column of porous particles, the gas will fill the pores in the particles. Thus, when the mass of the gas flow rate is measured at the exit of the column, it will be representative of flow only in the spaces between the particles because the mass of gas in the pores remains constant and stagnant. Further, the mass of the flowing gas will not be influenced by the shape of the particles as the gas will flow in the interstitial pore space whatever its shape happens to be. Therefore, the mass flow rate of the gas will be representative of the "impervious volume envelope" in the model.
3.1. Packing of Porous and Nonporous Irregularly Shaped Particles. Ergun next proceeds to test the process by carrying out experiments. The description that he gives of his experimental apparatus is minimal at best. He reports that "The apparatus consisted of a glass tube, two capillary flow-meters, and three manometers." He proceeds to describe in detail the technique by which he packed the glass tube with particles and the position of the manometers within the column which was the method he used to measure the pressure drops. However, he is silent on how he used the capillary flow-meters to record gas flow rate. Since it is not possible to position the capillary flow-meters inside the column, the only place that his equation is valid, they must have been deployed outside the column where his equation is not valid. It is, therefore, surprising that he provides no details on what flow parameter (mass or volumetric) or what technique he used to capture the flow rate information outside the column and the basis upon which he related such measurements to the pressure drop measurements taken inside the column.

His column packing technique involved the packing of a known mass of particles of narrow particle size distribution by using an upward flow of gas to expand the packed bed. A vibrator was also used to agitate the particles while forming the packed bed structure. The flow rate of the upflow of gas will determine the degree of bed expansion and thus is an efficient way to vary the external porosity of the column. When the vibrator and gas up-flow are turned off the bed formed has a fixed porosity. The measurements of pressure drop and gas flow rate were taken in the opposite direction of gas flow, that is, in a downward direction. This prevented the bed from expanding under the flow of gas and thus all measurements represented that of a fixed bed. Each particle size fraction was packed to between 6 and 12 different external porosities. Ergun then compares the results for calculated solid particle density using both versions of the equations with both porous and nonporous particles. In addition, he compares his results for his nonporous particles to conventional wet immersion techniques and concludes that they compare favorably.

Ergun's data consists of three distinct categories of measurements. They are as follows.

(a) Table I: Particle Densities of Crushed Nonporous Solids. In this category of data, Ergun shows his measurements and solid particle density determinations of 4 different particle size fractions of pulverized glass and river sand. He also displays the results obtained by the conventional technique of water displacement. We note that, in all cases, the values obtained by the water displacement technique correspond well to the values obtained by Ergun via his (5). Ergun's Figure 1 displays his data for the 80-100 mesh Brown River Sand contained in this category of particle definition (nonporous solids).

(b) Table II: Particle Density Determinations for High Temperature Oven Coke. This category of data contains the results of measurements of nine different particle size fractions of high temperature oven coke 
which represents columns packed with porous particles. Ergun's Figure 2 contains data from this category having a particle size distribution of 8-16 mesh.

(c) Table: IV Particle Density Determinations of Cokes of 40- to 60-Mesh Size. This category of data is again all porous particles but this time having the same particle size fraction of 40-60 mesh but having a different origin and thus various solid particle densities. Ergun's Figure 3 data is taken from this category but does not specify which individual coke designation it represents.

In this paper, Ergun deals with both nonporous and porous particles. His experimental protocol is designed to develop a methodology to identify the external porosity of a packed column of porous particles. The external porosity of a packed column, represented by $\varepsilon_{0}$ in our definition of terms, is the volume of the free space between the particles expressed as a fraction of the total volume of free space in the empty column. Because, however, one cannot distinguish between the free space between the particles and the free space within the pores of porous particles, a determination of the external porosity is difficult in columns packed with porous particles.

Ergun is attempting to exploit his newly developed pressure drop/fluid flow equation by back-calculating for the single unknown column external porosity. His methodology is based upon the principle that if one knows all the variables in the equation except one and one knows the product of all the variables, that is, the pressure drop, one can backcalculate for the unknown parameter. Accordingly, he first attempts to validate his concept by using nonporous particles of known solid particle density and comparing his results to the other independent method by which the original result was obtained. This information is contained in his Table I.

3.2. Contradictory Statements/Definitions. Ergun uses his 1949 equation developed with Orning as the starting point for his methodology. However, he introduces it in this paper (1951) as his (1) in which he defines the velocity term, $U_{m}$, as "the linear gas velocity based on the cross section of the empty column." This definition we recognize simply as the superficial velocity of the column based upon the usual differential pressure drop across the column, $\Delta P$. This newly minted definition is in direct contradiction to his definition of the velocity term in his original development in which it was defined by himself and Orning as $u_{m}$, which they defined as "the superficial velocity at the mean pressure." Accordingly, it appears that Ergun is now overtly equating the superficial velocity at the recorded pressure drop across the column, $\Delta P$, to the superficial velocity of the average (mean) of the pressure at the entrance and exit of the column, $\Delta P / 2$.

Moreover, in commenting on his experimental results in his Figures 1 and 2 in the main body of his paper, Ergun states: "When the ratios of pressure gradient to average velocity are plotted against mass flow rate according to (1), a straight line is obtained (his Figures 1 and 2) having intercept $a$ and slope $b$." The problem here is that his Figures 1 and 2 have their abscissa terms labeled as "flow rate at STP" with the designated units of cc./sec. which is a unit of volumetric flow rate not mass flow rate. It would appear then that Ergun is using some hodgepodge of parameters and definitions which would appear to confuse mass flow rate with volumetric flow rate, on the one hand, and superficial velocity at the mean pressure with superficial velocity at STP, on the other hand.

3.3. The Mysterious Multiplier $\left(5.79 \times 10^{3}\right)$. In the caption underlying his Figures 1 and 2 of the 1951 paper, Ergun asserts that a multiplier of 5,790 must be used to connect his data displayed in the figures to his data listed in his Table I. However, he is silent on the reason for such an enormous correlation multiple and does not give even a scintilla of hint as to what it represents. Undoubtedly, the value of 5,790 results from the exact experimental protocol which he was using to measure the flow properties of the fluid in his experiments. Characteristically, though, he is silent on the details of how and what he measured. For instance, he states that he used "two capillary flow-meters, and three manometers." We wonder why the need for two capillary flow-meters. Perhaps one was for volumetric data and one for gravimetric? He describes the reason for using two manometers, that is, at two different bed heights within the column, but we wonder what the third manometer was for! Perhaps it was for measuring the pressure outside the column? Regardless, however, of what particular combinations of experimental apparatus and protocols Ergun used to define his multiplier, it is certain that they were aimed at accounting for the effect of fluid (gas) density, a topic which Ergun was careful to avoid in the main body of his paper. In fact, there is not even a single mention of the need to consider the compressibility of the fluid (gas) in this entire paper of 1951.

We have determined that the mysterious multiplier, $M_{m}=$ 5,790 , was the ratio of the slopes of the straight lines obtained in the plots of his measured data. Ergun was, apparently, measuring both volumetric flow rate and mass flow rate at the column exit and by taking the ratio of the slopes of the straight lines obtained he generated his mysterious multiplier of 5790 which he could use to relate the two results. This explains the need for the two capillary flow-meters. Moreover, although Ergun was measuring both volumetric and mass flow rate, he chose to report only the results of his mass flow rate measurements in the many tables of this 1951 paper. In other words, he was measuring the equation of the line generated by his theoretical equation for volumetric flow rate which has the format, $y=a+b q^{\text {stp }} / A$, and he was also measuring the mass flow rate, which has the format, $y=a+b q_{m}{ }^{\text {stp }} / A$, but he chose to report only the latter, his values for mass flow rate. In addition, he also used the same symbols, $a$ and $b$, to represent the intercept and slope of the line, in either case, once in the 1949 paper when he was referring to the mass flow rate and once in his 1951 paper when he was referring to the volumetric flow rate. Although both equations of the line have the same value of intercept, $a$, they do not have the same slope $b$. Since, however, he presented his measured data in graphical form as volumetric flow rate in this 1951 paper (see his Figure 1), which has volumetric flow rate at STP on the abscissa, and reported his results as mass flow rate in the tables, he had to use this mysterious multiplier as a conversion factor between the two formats. Accordingly, we know that the mysterious 
multiplier is the ratio of the column cross-sectional area to the density of the gas at STP, $A / \rho_{f}{ }^{\text {stp }}$.

\section{The 1952 Paper}

In his 1952 paper, Ergun builds upon developments in his two previous papers. He begins his 1952 paper with the postulate that "pressure losses are caused by simultaneous kinetic and viscous energy losses" and concludes that "the following comprehensive equation is applicable to all types of flow." Consider

$$
\frac{\Delta P g_{c}}{L}=\frac{150(1-\varepsilon)^{2} \mu U_{m}}{\varepsilon^{3} D_{p}{ }^{2}}+\frac{1.75(1-\varepsilon) G U_{m}}{\varepsilon^{3} D_{p}},
$$

where $\Delta P=$ pressure loss in force units, $g_{c}=$ gravitational constant, $\varepsilon=$ fractional void volume in bed, $\mu=$ absolute viscosity of fluid, $U_{m}=$ superficial fluid velocity measured at average pressure, $G=$ mass flow rate of fluid, $L=$ height of bed, and $D_{p}=$ average spherical diameter equivalent of particle.

Ergun states that the purpose of this paper is to verify empirically an earlier theoretical development and that the new experimental work involved gas flow through crushed porous solids. He underscores the fact that this is in contrast to many previous studies in which the experiments involved nonporous solids. He goes on to say that most authorities agree that the most important factors to be considered are

(1) rate of fluid flow,

(2) viscosity and density of fluid,

(3) closeness and orientation of packing,

(4) size, shape, and surface of particles.

Ergun notes that a linear relationship exists between $f_{v}$, the viscous friction factor, and $R_{e p} /\left(1-\varepsilon_{0}\right)$, which is otherwise known as the modified Reynolds number. Utilizing a fitting algorithm of least squares to his measured $\Delta P$ data, he determines from 640 experiments the empirical values of $k_{1}$ and $k_{2}$. The data set involved various sized spheres, sand, pulverized coke, and the following gases: $\mathrm{CO}_{2}, \mathrm{~N}_{2}, \mathrm{CH}_{4}$, and $\mathrm{H}_{2}$. Thus, the particles used in the study involved both porous and nomporous solids, whereas the fluids used in all cases were compressible gases. The values for $k_{1}$ and $k_{2}$ which Ergun claims were generated by his experiments are 150 and 1.75 , respectively. Substituting 150 and 1.75 for the values of $k_{1}$ and $k_{2}$, respectively, Ergun goes on to express his equation as

$$
f_{v}=150+\frac{1.75 R_{e p}{ }^{m}}{\left(1-\varepsilon_{0}\right)} .
$$

This is a representation in which the viscous energy losses have been normalized. tion is

He suggests that another form of representing the equa-

$$
f_{k}=\frac{150\left(1-\varepsilon_{0}\right)}{R_{e p}^{m}}+1.75 .
$$

This is a representation in which the kinetic energy losses have been normalized.
4.1. Ergun's Presentation of His Measured Data. Figure 1 in this 1952 paper is a plot of $\Delta P / L \mu_{s}$ versus $q_{m}$. In this graphical representation of his measured data, Ergun focuses on the linearity of the relationship. Although the quantities plotted on both axes supposedly represent his measured values and are not dimensionless, Ergun does not specify the units of measure. This is a critical omission, especially in a paper designed to validate a theoretical model. Figure 2 in his paper contains two plots which show the influence of column porosity in both viscous and kinetic terms of his equation. Ergun points out that since both plots are straight lines which pass through the origin, this demonstrates that the respective porosity dependence terms for the viscous and kinetic energy losses are correct. In his Figure 3, Ergun simply plots his results from additional experiments carried out with a different particle size and with different bed porosities. Unlike his data in his Figure 1, however, he does not provide the underlying packed column densities, which are necessary to independently evaluate this plot. The fact, though, that the plot is also a straight line which passes through the origin suggests that the viscous porosity dependence term is universal in nature. The plot in his Figure 4 is a different representation of Ergun's measured data than that displayed in his Figure 1. It is unclear exactly what the significance of this plot is, other than condensing into one plot both of his plots in his Figure 2, since it results in values for the intercept and slope identical to those derived in the plots in his Figure 2. Again, no units of measure are specified.

Thus, Ergun made a significant contribution to the understanding of the pressure/flow relationship in granular beds by recognizing the additive nature of viscous and kinetic energy losses to the overall pressure drop. This advancement in understanding enabled him to establish two versions of a friction factor, each of which could be used for normalizing either kinetic or viscous energy losses.

\section{Anatomy of the Ergun Equation}

The Ergun equation is based upon a basic principle annunciated by Ergun and Orning in their 1949 publication which they expressed as follows: "The ratio of pressure gradient to superficial fluid velocity in packed columns is shown to be a linear function of fluid mass flow rate." Based upon this a priori notion, they developed their equation which established a relationship between fluid flow rate and differential pressure across a packed column. We repeat here that equation from their 1949 paper:

$$
\frac{d P}{d L}=\frac{2 \alpha\left(1-\varepsilon_{0}\right)^{2} S_{v}{ }^{2} \mu_{s} \eta}{\varepsilon_{0}{ }^{3}}+\frac{\beta\left(1-\varepsilon_{0}\right) S_{v} \mu_{s}{ }^{2} \rho_{f}}{8 \varepsilon_{0}{ }^{3}} .
$$

The authors embedded into the equation the term $2 \alpha$ on the viscous side and the term $\beta / 8$ on the kinetic side based upon a theoretical postulate which arose out of two concepts. One of the concepts was based upon the notion of cylindrical channels which contributed the 2 to the viscous term and the $1 / 8$ to the kinetic term. The other concept had to do with the notion of sinuous flow between the particles in the packed column and contributed the $\alpha$ to the viscous side 
of the equation and the $\beta$ to the kinetic side. Since we do not accept the rationale underlying either concept for the reasons we pointed out above, in the first instance, and since it complicates our uncovering of the correct values for the coefficients $k_{1}$ and $k_{2}$, in the second instance, we will now undo these theoretical developments and restate the equation by recombining these terms into the residual coefficients $k_{1}$ and $k_{2}$. Thus we may write

$$
\frac{d P}{d L}=\frac{k_{1}\left(1-\varepsilon_{0}\right)^{2} S_{v}{ }^{2} \mu_{s} \eta}{\varepsilon_{0}{ }^{3}}+\frac{k_{2}\left(1-\varepsilon_{0}\right) S_{v} \mu_{s}^{2} \rho_{f}}{\varepsilon_{0}{ }^{3}} .
$$

Next, we substitute for $d_{p}=6 / S_{v}$ and $d P / d L=\Delta P / L$, which gives

$$
\frac{\Delta P}{L}=\frac{36 k_{1}\left(1-\varepsilon_{0}\right)^{2} \mu_{s} \eta}{\varepsilon_{0}{ }^{3} d_{p}{ }^{2}}+\frac{6 k_{2}\left(1-\varepsilon_{0}\right) \mu_{s}^{2} \rho_{f}}{\varepsilon_{0}^{3} d_{p}} .
$$

Recombining the 36 into $k_{1}$ and the 6 into $k_{2}$ gives

$$
\frac{\Delta P}{L}=\frac{k_{1}\left(1-\varepsilon_{0}\right)^{2} \mu_{s} \eta}{\varepsilon_{0}{ }^{3} d_{p}^{2}}+\frac{k_{2}\left(1-\varepsilon_{0}\right) \mu_{s}^{2} \rho_{f}}{\varepsilon_{0}^{3} d_{p}},
$$

where $k_{1}$ and $k_{2}$ are the residual components of viscous and kinetic contributions, respectively, embedded in the measured instantaneous pressure drop, $\Delta P$, which Ergun and Orning's theoretical development did not account for in their final equation. We point out, however, that there is nothing fundamentally "constant" about the residual coefficients $k_{1}$ and $k_{2}$. They are simply the remainder of the unaccounted for contributions, but assuming that the equation is valid in the first place, the value of this remainder should always be the same after all variables are taken into account, and, thus, it is in this context that we refer to them as being "constant."

5.1. The 1949 Columns with Spherical Nonporous Particles. In the 1949 paper, Ergun and Orning introduced their newly minted equation along with a significant amount of empirical data. They present their results in graphical form in three plots of data for packed columns which are linear, Figures 1,2 , and 3 . We note that they measured the flow rate of the fluid outside the column. "Gas flow was measured with a series of capillary flow-meters, each calibrated against wettest meters." We therefore know that the values which they used for mass flow rate displayed on the abscissa of the graphs, at least in their own plots, are representative of measurements taken at standard temperature and pressure (STP).

On the other hand, we know that they measured the pressure drops inside the column. "Pressure drop measurements were made in a glass tube, 1 inch in inside diameter and 30 inches long, fitted with a fritted-glass disc to obtain uniform gas flow at the bottom and with pressure taps at the top and at a point just above the fritted-glass disc." Conspicuously absent from their measurements, however, is any data or mention of the independent variable of "superficial velocity at the mean pressure" which appears in their equation. Additionally, their description of the experimental apparatus used gives no hint of how one would even measure this parameter. It is also significant that, instead of reporting their measurements of all independent variables on an individual variable basis, a customary practice, the authors made only a partial reporting in the form of their pressure drop and flow rate measurements displayed in their Figure 1. Since their Figure 1 plot, however, gives only the ratio of pressure drop and velocity multiplied by column length, it is ambiguous with respect to the embedded values of $k_{1}$ and $k_{2}$ because the values of all the equation variables have not been identified.

We conclude, therefore, that Ergun/Orning measured the pressure generated inside a packed column while passing a fluid (gas) through the column. A gas is compressible and therefore its density is a function of the pressure at which it is measured. Even if one measures the mass flow rate outside the column, however, one still has to know the density of the fluid inside the column in order to calculate the volumetric flow rate inside the column, because it is this volumetric flow rate that underlies the calculation of superficial fluid velocity which, in turn, is called for in both the viscous and kinetic terms of the authors' pressure/flow equation.

5.2. A Closer Look at the Experimental Data. We begin our analysis by generating our own spreadsheet of calculations underlying author's Figure 1. The data underlying their other plots, for example, Figures 2 and 3, were taken from the literature and they did not report all the appropriate underlying independent variables in the paper, so we neglect these data sets in our analysis.

To begin, we define here the basis upon which we derive the values of $\alpha$ and $\beta$ which appears in author's Table 1. We can show our methodology by first looking to authors' (7):

$$
\frac{\Delta P}{L \mu^{m}}=\frac{2 \alpha\left(1-\varepsilon_{0}\right)^{2} S_{v}{ }^{2} \eta}{\varepsilon_{0}{ }^{3}}+\frac{\beta\left(1-\varepsilon_{0}\right) S_{v}\left(q_{m} / A\right)}{8 \varepsilon_{0}{ }^{3}} .
$$

It follows from the plot in authors' Figure 1 which is a plot of the left hand side of this equation versus the quantity $\left(q_{m} / A\right)$ on the right hand side that the values of $\alpha$ and $\beta$ are determined thusly:

$$
\begin{gathered}
\alpha=\frac{[\text { Intercept }] \varepsilon_{0}{ }^{3}}{2\left(1-\varepsilon_{0}\right)^{2} S_{v}{ }^{2} \eta}, \\
\beta=\frac{[\text { Slope }] 8 \varepsilon_{0}{ }^{3}}{\left(1-\varepsilon_{0}\right) S_{v}},
\end{gathered}
$$

where [Intercept] and [Slope] represent the intercept and slope of the line in their Figure 1, respectively. These calculations are displayed in our Table 1 for the data corresponding to the columns in the author's Figure 1 which were tested with nitrogen: lead shot with particle size of $0.497 \mathrm{~mm}$ (our column A), glass beads with particle size $0.570 \mathrm{~mm}$ (our column B), and, finally, lead shot with particle size $0.562 \mathrm{~mm}$ (our column C). The particles in these columns were totally smooth, spherical in shape, and nonporous, exactly what would be generally accepted in the field as "known standards." This results from the fact that the physical characteristics of these particles can be well characterized by various independent techniques and there exists no doubt about either surface 
area or solid particle density, two physical properties which are necessary in Ergun's calculations underlying his equation. It will be appreciated that Ergun did not measure the particle size as one might do in these days of sophisticated analytical instrumentation. Rather, he measured the surface area of the particles $\left(S_{p}\right)$ by nitrogen absorption, or the equivalent, and calculated the average particle size as described in his paper $\left(d_{p}=6 / S_{v}\right)$. In addition, the value of the solid particle density is used to calculate the column porosity, $\varepsilon_{o}$, another parameter specified in the equation.

Ergun and Orning imported into their (7) the numerical values of 2 in the viscous term and $1 / 8$ in the kinetic term and the variable parameters of $\alpha$ and $\beta$ also in the viscous and kinetic terms, respectively. These additions to their equation were based upon their own theoretical postulates and, as we demonstrated above, represent a hodge-podge of commingled variables. Accordingly, as we explained above, the values of $k_{1}$ and $k_{2}$ can be identified as follows:

$$
\begin{aligned}
& k_{1}=\frac{[\text { Intercept }] \varepsilon_{0}{ }^{3} d_{p}{ }^{2}}{\left(1-\varepsilon_{0}\right)^{2} \eta}, \\
& k_{2}=\frac{[\text { Slope }] \varepsilon_{0}{ }^{3} d_{p}}{\left(1-\varepsilon_{0}\right)} .
\end{aligned}
$$

In addition to the values for $\alpha$ and $\beta$, our Table 1 also displays the values of $k_{1}$ and $k_{2}$. We can see from our Table 1 , therefore, that column A has the values of 2.0 and 2.7 for $\alpha$ and $\beta$ as specified in Table 1 of Ergun's paper and the corresponding values of 145 and 2.01 for $k_{1}$ and $k_{2}$, respectively. Similarly, our column B has the values of 2.0 and 2.8 for $\alpha$ and $\beta$ with corresponding values for $k_{1}$ and $k_{2}$ of 141 and 2.06, and, finally, our column $C$ has the values of 1.9 and 2.7 for $\alpha$ and $\beta$ with corresponding values for $k_{1}$ and $k_{2}$ of 134 and 2.03. Finally, we emphasize here that if Ergun and Orning had used an incompressible fluid (liquid) to make their flow measurements at the column exit, their measurements would have been accurate in an absolute sense and these values for $k_{1}$ and $k_{2}$ would be the correct values. However, this was not the case since the authors used nitrogen gas as the fluid of choice.

In our Figure 1(A1) we show a plot of the results which the authors reported for column A in their Figure 1 of the 1949 paper. Note that we use the symbol of $\mu_{s}$ stp for the superficial velocity on the ordinate of our plot. This correctly represents the superficial velocity which the authors actually measured at STP conditions outside the column. This is in contrast to the symbol $U_{m}$ which the authors used in the ordinate of their plot and for which they provided no definition within the four corners of the 1949 paper. It is particularly relevant to point out that in their glossary of terms at the end of the paper the authors did provide a definition and symbol, $u_{m}$, for the superficial velocity at the mean pressure. However, they did not use that symbol on the ordinate of the plot and they never mentioned any methodology that could be remotely connected to measuring the superficial velocity at the mean pressure, in the main body of the paper. In our Figure 1(A1), we can see that the equation of the line produces a slope and intercept with values of 616 and 96, respectively, for column A. Similarly, in our Figure 1(A2), we see that the corresponding values for column B are 675 and 90, and, in our Figure 1(A3) for column C, the values are 536 and 68.

5.3. Correcting the Reported Data. In order to extract the correct values for the coefficients, $k_{1}$ and $k_{2}$ in Ergun and Orning's equation from their measured data, we have but one choice. That choice is to use a residual mentality. By this we mean that we must identify the correct value of all variables in the equation at the particular moment that the pressure drop was measured and by inserting these measured values into the equation, we can then use the equation relationship between measured pressure drop and column and fluid variables to back-calculate for the residual coefficients, $k_{1}$ and $k_{2}$. Ergun and Orning relied upon the properties of a straight line, the intercept, and slope, to report their measured data. This is an ambiguous methodology with which to report data when all the individual values of the equation parameters are not separately identified. Because they did not report measurements for many of the variables, for example, temperature, fluid viscosity, fluid density, and fluid superficial velocity at the mean pressure, we cannot rely, in our analysis, on this ambiguous presentation of their reported data shown in Figure 1 alone. In addition, they only reported a nominal value for the particle surface area, shown in Table 1 of their paper, and solid particle density reported in the experimental section of the paper and, in addition, completely ignored the column length which is also an important equation parameter. Accordingly, their reported values for column porosities were also nominal, since they were based upon these other nominal values for the parameters upon which the calculations were based. These nominal values are not sufficiently accurate to identify precisely the residual coefficients $k_{1}$ and $k_{2}$. This is because the author's equation has a porosity dependence term, ( 1 $\left.\left.\varepsilon_{0}\right) / e_{0}{ }^{3}\right)$, and a particle size dependence term, $d_{p}{ }^{2}$, which are extremely sensitive to small discrepancies and, as a result, any inaccuracies in the measured values of these two critical independent variables will have a huge impact on the value of the residual coefficients in this process. It will be appreciated, for instance, that the porosity dependence term alone is sensitive to the fourth power of the column external porosity $\varepsilon_{0}$ (to a first approximation) which is one of the most sensitive relationships in all of nature.

Accordingly, we are left to deal with the inadequacies of the author's experimental protocol, the lack of critical unreported variables, and the ambiguous manner in which they presented their measured data. We begin by inputting into our spreadsheet of calculations in our Table 1 all the pertinent information underlying author's Figure 1. The author's equation, assumed to be valid in this extraction process, represents a closed system and must, therefore, obey the laws of thermodynamics, which dictates that we include in our calculations a provision for the fact that both fluid viscosity and fluid density are, individually, a function of temperature, and fluid density, in addition, is also a function of operating pressure. Consequently, in our spreadsheet, we use a combination of Sutherland's formula for estimating the fluid viscosity of nitrogen gas at any given temperature and 


\begin{tabular}{|c|c|c|c|c|c|c|c|c|c|}
\hline & 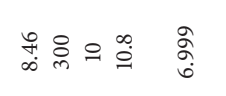 & 䒽 & 浐 & 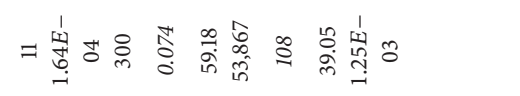 & 商路 & 高 & $\cong$ & $\stackrel{8}{i}$ & $\stackrel{9}{2}$ \\
\hline & 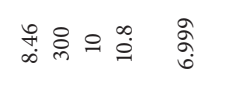 & 菺 & 总 & 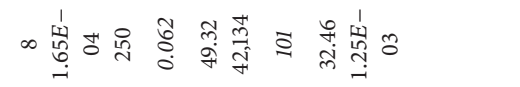 & 离 8 & 高 & $\ddot{g}$ & $\stackrel{\text { \%े }}{\mathrm{i}}$ & 2 \\
\hline 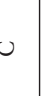 & 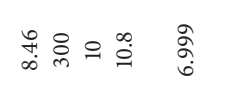 & 㡈 & $\stackrel{0}{:}$ & 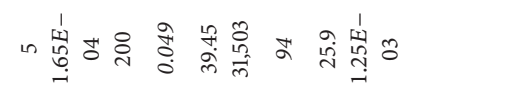 & 嵌 & 㝵 & $\stackrel{n}{9}$ & $\stackrel{\text { ले }}{\mathrm{i}}$ & 2 \\
\hline$v$ & 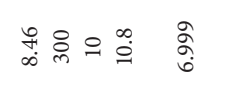 & 蒿 & 兽 & 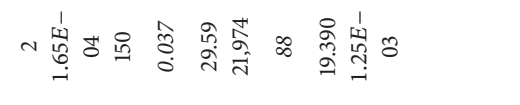 & 蚂 & 参 & $\dddot{m}$ & $\stackrel{\text { ì }}{\mathrm{i}}$ & 9 \\
\hline p & 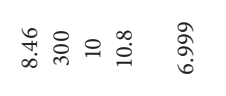 & 菺 & : & 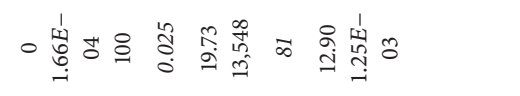 & 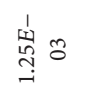 & 熟 & $\ddot{g}$ & $\stackrel{8}{\text { S }}$ & 2 \\
\hline v & 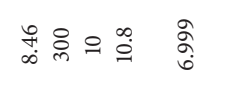 & 撂 & 器 & 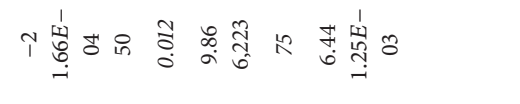 & 湈号 & 高 & $\overleftrightarrow{\cong}$ & 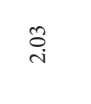 & 9 \\
\hline v & 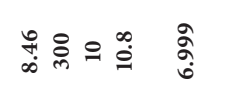 & 雚 & $\stackrel{\circ}{: 0}$ & 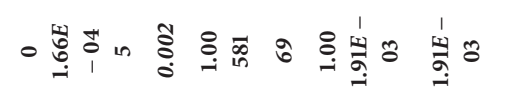 & & 颉 & $\stackrel{ \pm}{ \pm}$ & $\cong$ & 9 \\
\hline a & 员웍국 & 商 & 鉫 & 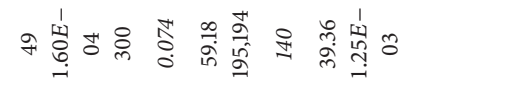 & 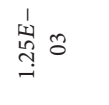 & 㠓 & 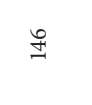 & $\stackrel{\circ}{\sim}$ & i \\
\hline D & 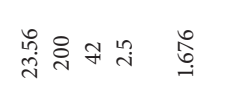 & 尊 & 高 & 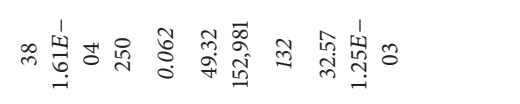 & 嵌 & 㝵 & 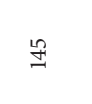 & $\stackrel{\circ}{\text { i̊ }}$ & $\stackrel{i}{i}$ \\
\hline 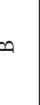 & 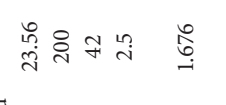 & 量 & 营 & 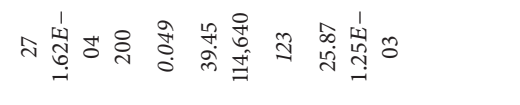 & 嵌密 & 紡 & \pm & $\stackrel{\overbrace{}}{\stackrel{\sim}{i}}$ & $\stackrel{i}{i}$ \\
\hline & 等 & 高 & $\stackrel{\substack{0 \\
0}}{2}$ & 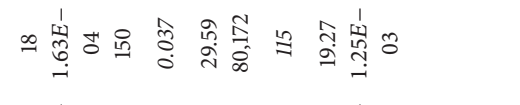 & 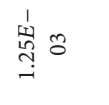 & 总 & $\mathscr{I}$ & $\stackrel{\circ}{\stackrel{\leftrightarrow}{i}}$ & $\stackrel{i}{i}$ \\
\hline$\infty$ & 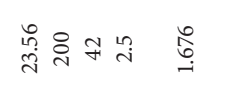 & 产 & 突 & 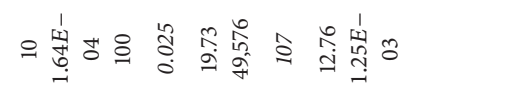 & 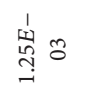 & 营 & $\mp$ & $\stackrel{\circ}{i}$ & $\stackrel{i}{i}$ \\
\hline a & 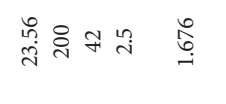 & 兽 & $\stackrel{\substack{3 \\
0}}{0}$ & 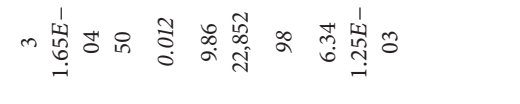 & 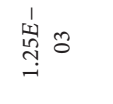 & 敦 & $\exists$ & 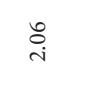 & $\stackrel{i}{i}$ \\
\hline$a$ & 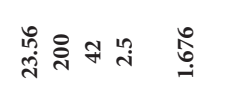 & 晜 & 营 & 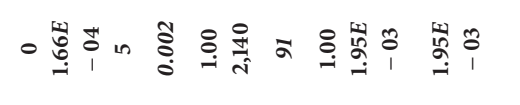 & & 兽 & $\exists$ & $\stackrel{\widetilde{3}}{3}$ & $\stackrel{i}{i}$ \\
\hline 4 & 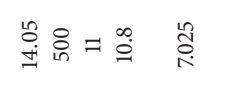 & 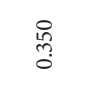 & $\frac{0}{8}$ & 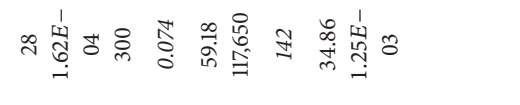 & & 㝵 & $\stackrel{\infty}{ \pm}$ & $\vec{i}$ & \\
\hline 4 & 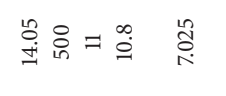 & 品 & $\stackrel{\circ}{:}$ & 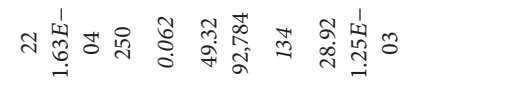 & 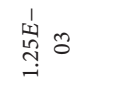 & 高 & $\Xi$ & $\vec{i}$ & $\stackrel{i}{i}$ \\
\hline 4 & 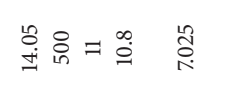 & 总 & $\stackrel{\circ}{\circ}$ & 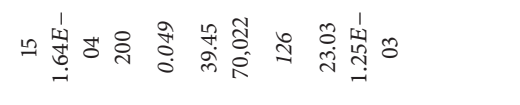 & 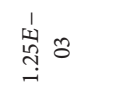 & 高 & $\dddot{q}$ & $\vec{i}$ & $\stackrel{i}{i}$ \\
\hline 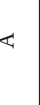 & 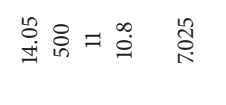 & 总 & 兽 & 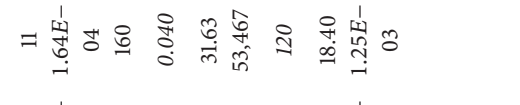 & 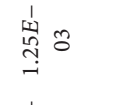 & 㝵 & $\nsubseteq$ & $\stackrel{\vec{i}}{i}$ & $\stackrel{\circ}{i}$ \\
\hline द & 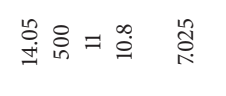 & 总 & 总 & 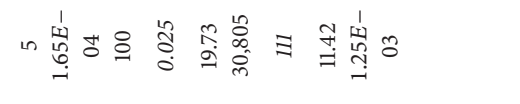 & 窎务 & 絭 & $q$ & $\underset{i}{i}$ & $\stackrel{i}{i}$ \\
\hline & 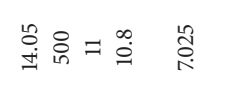 & 总 & $\stackrel{\circ}{\circ}$ & 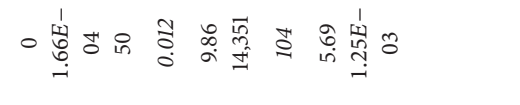 & 商 & 高 & 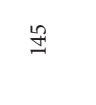 & $\underset{i}{i}$ & $\stackrel{\circ}{i}$ \\
\hline & 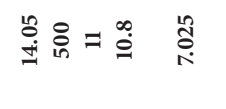 & 高 & 总 & 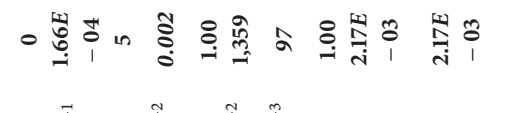 & & 我 & 9 & & $\stackrel{\circ}{i}$ \\
\hline & 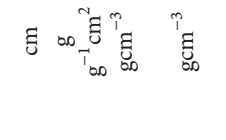 & 台 & & 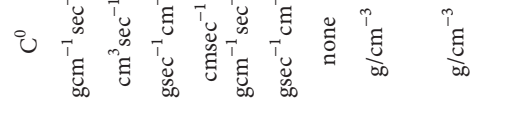 & ${ }^{i} \bar{E}$ & $\begin{array}{l}{ }^{n} \bar{E} \\
T_{\infty}^{5}\end{array}$ & 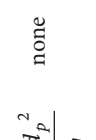 & $\stackrel{\check{\Xi}}{a}$ & $\stackrel{\varrho}{\check{E}}$ \\
\hline
\end{tabular}




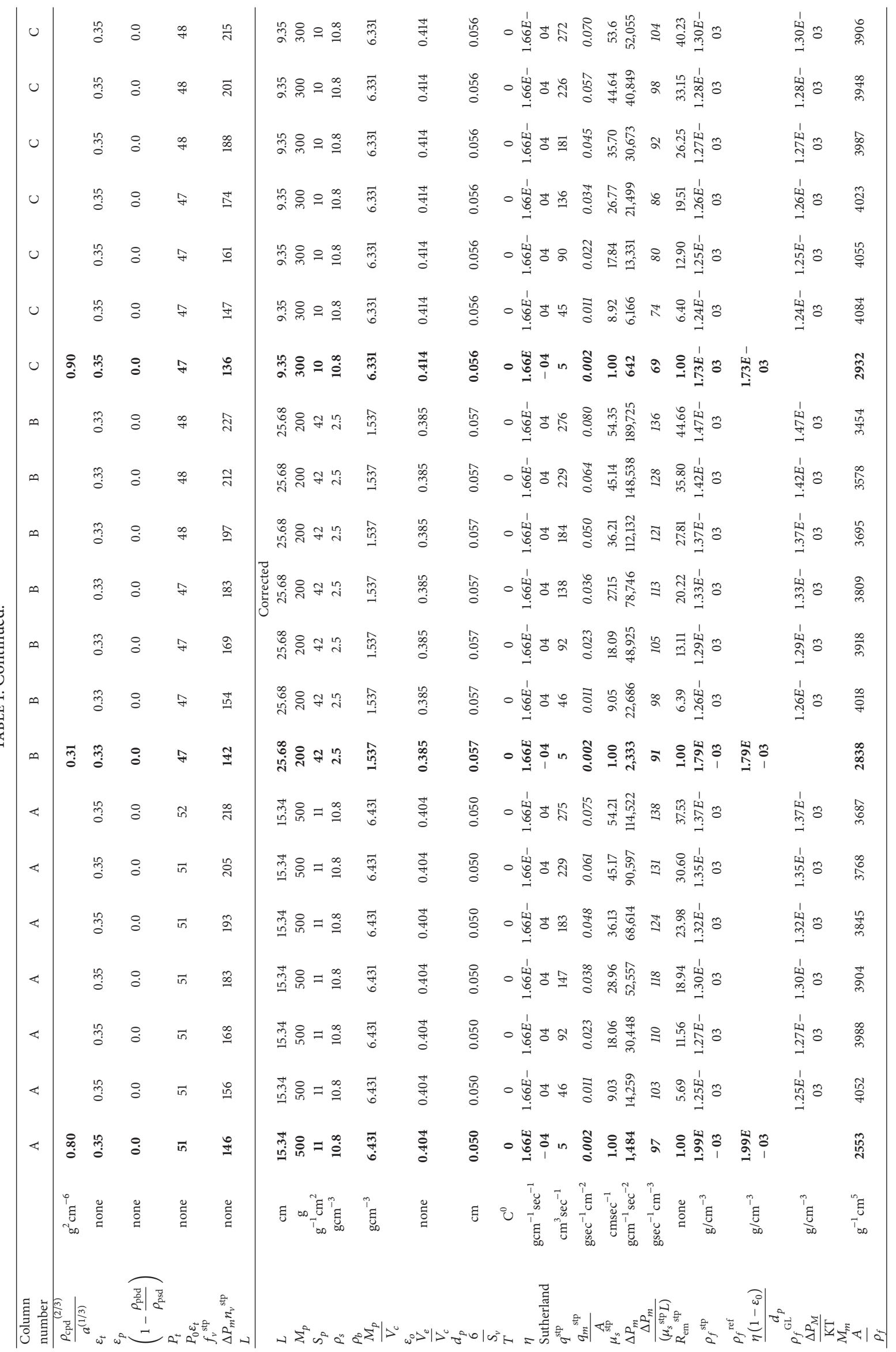




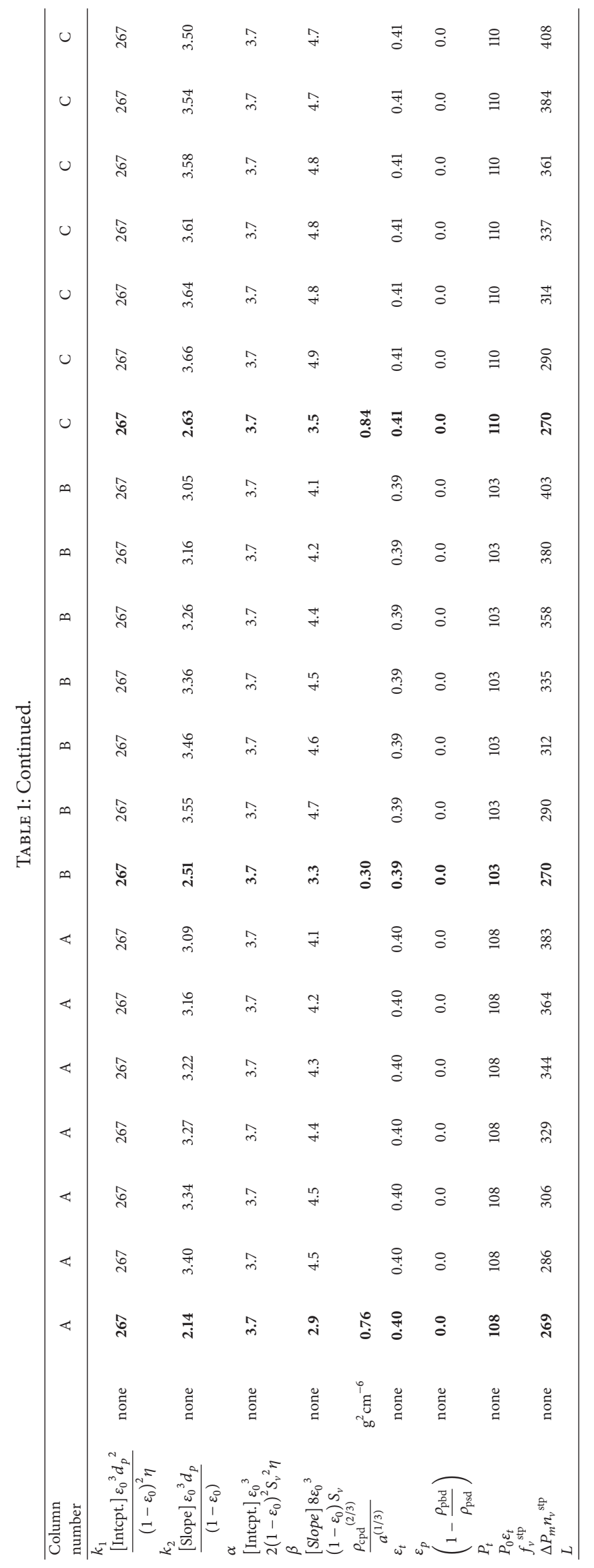


Ambiguously Reported Data-column A

Plotted according to Ergun's (7)

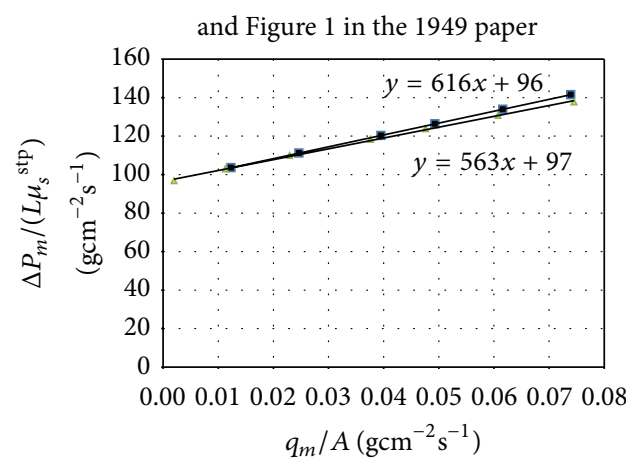

- Col. A reported — Linear (col. A reported)

$\Delta$ Col. A corrected — Linear (col. A corrected)

(A1)

Ambiguously Reported Data-column B

Plotted according to Ergun's (7)

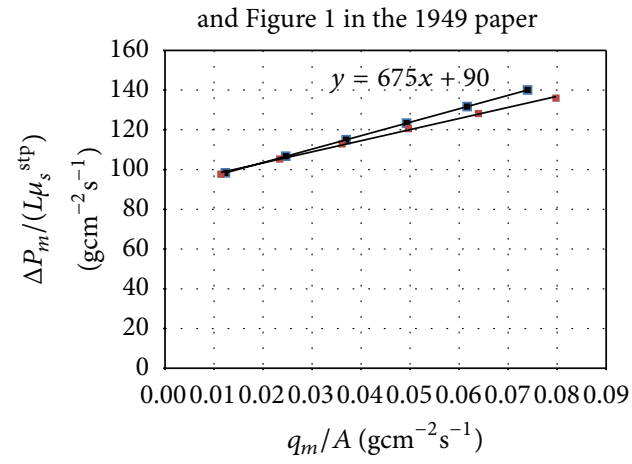

- Col. B reported L Linear (col. B reported)

- Col. B corrected — Linear (col. B corrected)

(B1)

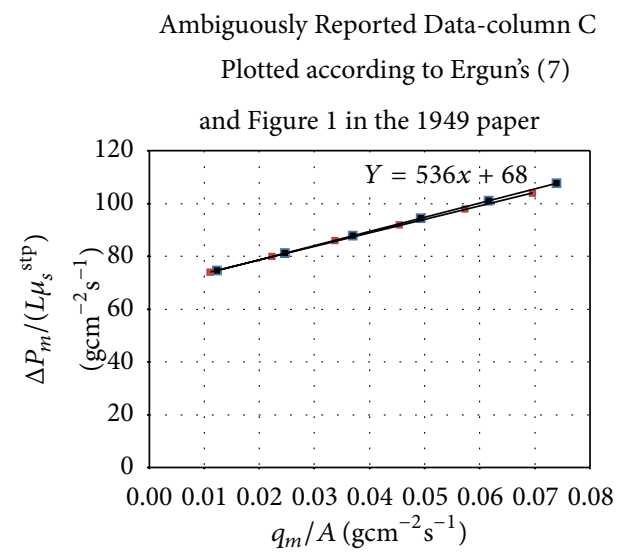

- Col. C reported L Linear (col. C reported)

- Col. C corrected — Linear (col. C corrected)

(C1)
Data reexpressed in dimensionless parameters-column A

Plotted according to Ergun's (13b)

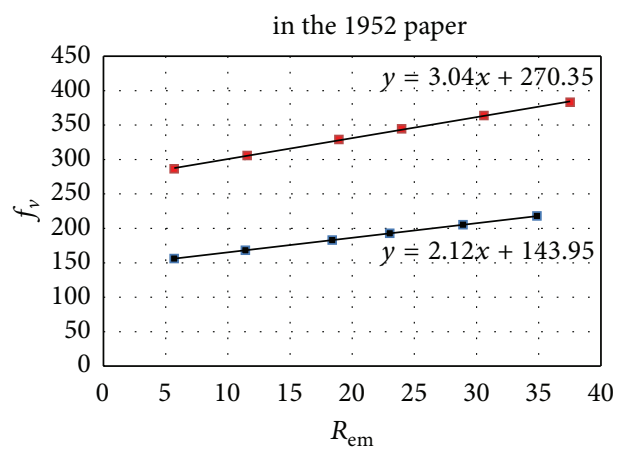

- Col. A reported — Linear (col. A reported)

- Col. A corrected — Linear (col. A corrected)

(A2)

Data reexpressed in dimensionless parameters-column B Plotted according to Ergun's (13b)

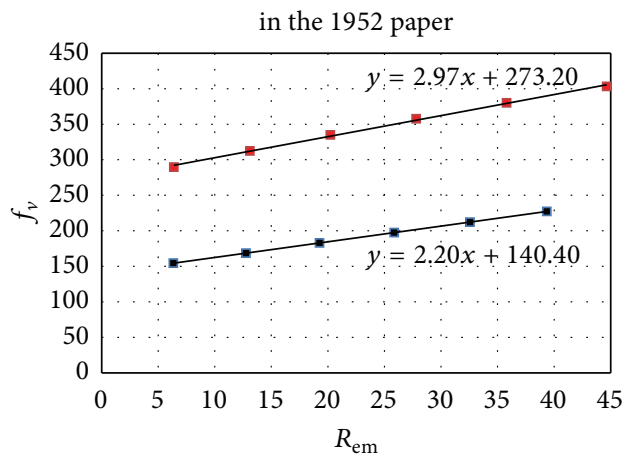

- Col. B reported L Linear (col. B reported)

- Col. B corrected — Linear (col. B corrected)

(B2)

Data reexpressed in dimensionless parameters-column C Plotted according to Ergun's (13b)

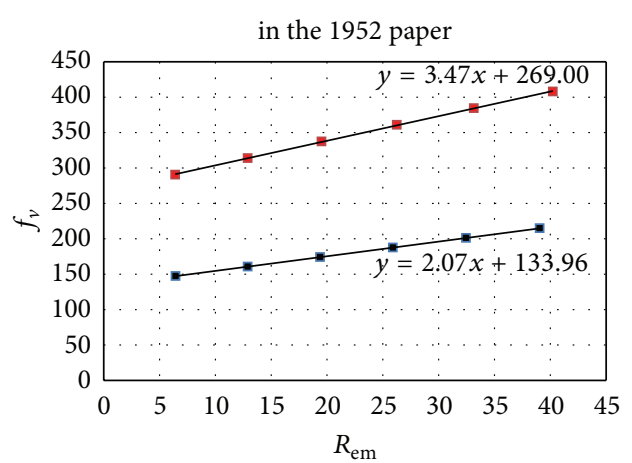

- Col. C reported L Linear (col. C reported)

- Col. C corrected L Linear (col. C corrected)

(C2)

(a)

FIgURE 1: Continued. 


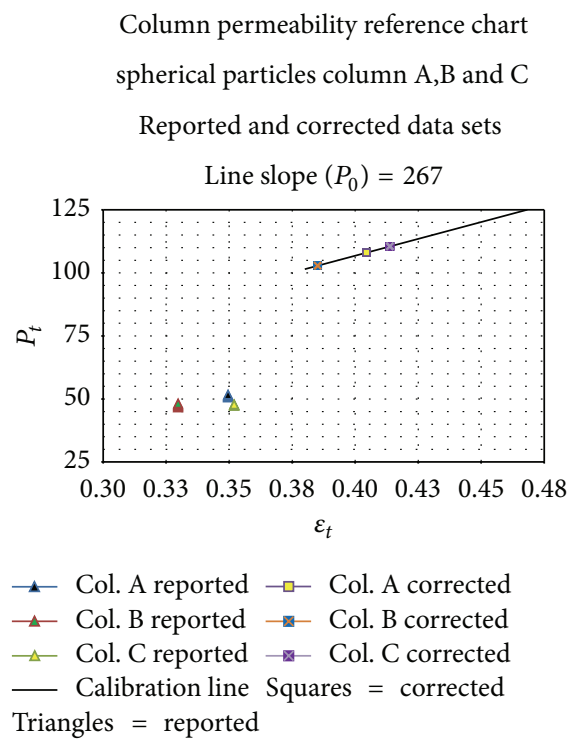

(D1)

(b)

Figure 1

the universal gas law to estimate the density of nitrogen as a function of both temperature and pressure. The authors did not specify any temperature values in their 1949 paper and, therefore, to be consistent with their 1951 and 1952 papers, we use the value of Ergun's mysterious multiplier equivalent, $A / \rho_{f}$, for the reported data in columns $\mathrm{A}, \mathrm{B}$, and C.

In our calibration procedure for each column, we impose a boundary condition inherent in the relationship between pressure drop and flow rate dictated by the Ergun equation. This boundary condition is born of the reality that the same compressible fluid when passed through two different columns at the same pressure drop will produce different flow rates depending on other variables of the column, for example, porosity and particle size. Therefore, each individual column must be calibrated with respect to these four independent variables, that is, fluid superficial velocity, fluid density, fluid viscosity, and column pressure drop. Looking to the relationships developed above, we can see that when the modified Reynolds number is unity, an important boundary condition regarding these column variables emerges. We begin by recalling the formula for the modified Reynolds number which is

$$
R_{\mathrm{em}}=\frac{\mu_{s} d_{p} \rho_{f}}{\eta\left(1-\varepsilon_{0}\right)} .
$$

Set a boundary condition that when $\mu_{s}=R_{\mathrm{em}}=1$.

It follows that

$$
\rho_{f}=\frac{\eta\left(1-\varepsilon_{0}\right)}{d_{p}} .
$$

The density of the fluid at this boundary condition is given by the expression $\eta\left(1-\varepsilon_{0}\right) / d_{p}$ which are all known (measureable) quantities. Accordingly, we can identify the sum of the values of $k_{1}$ and $k_{2}$ for each column at this boundary condition which is displayed in our Table 1 as the first data point listed for each column (when the modified Reynolds number is unity, $\left.f_{v}=f_{k}=\left(k_{1}+k_{2}\right)\right)$. In the data for the reported equation of the line, on the one hand, we use the mysterious multiplier equivalent value at each reported pressure drop. Therefore, we are acknowledging that the reported fluid velocity data is all taken at a fixed density, that is, the density at STP. This being the case, as dictated by the laws of thermodynamics, the reported data has a different temperature for each measured pressure drop within a given column and has varying values of temperature across all columns commensurate with the different resistance of each column. In our corrected data, on the other hand, we maintain a constant reference temperature of $0^{\circ} \mathrm{C}$ at each flow rate which is a more realistic scenario for what is happening within the packed column when the pressure drop measurements were taken. We show our corrected data for each of the columns A, B, and C as additional lines plotted alongside the reported data in our Figure 1(A1), Figure 1(A2), and Figure 1(A3). As can be seen from the plot, our corrected data for each of the columns A, B, and C has the same intercept as the reported data, but a different slope. Moreover, as seen in our Table 1, each of the three columns in the corrected data set yields a value close to 270 for the sum of the coefficients $k_{1}$ and $k_{2}$ at our boundary condition calibration point. This compares to a value of less than 150 for the reported data on each of the three columns.

In order to underline the shortcomings of the methodology chosen by the authors to represent their empirical data, we include another set of plots outlined in our Figure 1(A2), Figure 1(B2), and Figure 1(C2). This plot demonstrate the use of the parameters, $f_{v}$ and $R_{\text {em }}$, which Ergun introduced in his 1952 paper and which he used as the basis of his 
linear extrapolation (he called it least squares) in determining the values of 150 and 1.75 for the constants. Note that, in this frame of reference, the reported and corrected data sets yield straight lines which do not coincide and which, therefore, have entirely different slopes and intercepts. This plot demonstrates that when all the column variables are accurately identified the resultant values for $k_{1}$ and $k_{2}$ are totally different for the reported data set. Focusing now on the column porosity variable, in particular, since the authors provided no independently measured value for many of the underlying equation variables in their reported results, including the column length, $L$, the particle solid density, $\rho_{s}$, and the particle diameter, $d_{p}$, we have chosen to lump all the uncertainty in the values of these variables into the single value of the column length. This generates a value for the column porosity, $\varepsilon_{0}$, which is at odds with the values reported by the authors in the 1949 paper for this parameter. They reported values for the column porosity of $0.35,0.33$, and 0.352 for the columns $\mathrm{A}, \mathrm{B}$, and $\mathrm{C}$, respectively. To one of skills in the art, these values are too low for rigid spherical particles with narrow particle size distributions similar to what was used in this paper and are the result of the technique used by the authors to calculate this parameter. They did not measure the porosity directly. They calculated it from the particle surface area, particle solid density, and column length in conjunction with the mass of particles packed into each column. The discrepancy in the values of the reported column porosity is the cumulative effect of using nominal values instead of carefully measured individual values for each of these independent variables.

Before we examine in more detail the corrected values for the all-important column porosity parameter, $\varepsilon_{0}$, however, we necessarily digress here to point out another source of error in Ergun's methodology which is born of inaccurate thinking when it comes to fundamental definitions. The error we refer to relates, not surprisingly, to the measurement of fluid velocity in packed columns. Unlike the manner in which engineers calculate fluid velocity, by measuring the fluid flow rate and dividing by the cross-section of the column, chromatographers measure the time it takes for an injected solute to traverse the column length. This method identifies the fluid velocity as the rate of change of distance with time which is the true definition of velocity. Indeed, strictly speaking, fluid velocity has nothing to do with volumetric flow rate or column cross-section. However, although convenient for doing calculations, the latter engineer's methodology represents sloppy science which can lead to disastrous consequences. Indeed, we suggest that this was yet another contributing factor to Ergun's compounding of errors. As can be seen in the glossary of terms at the end of Ergun's 1952 paper, his definition of mass flow rate, $G$, is incorrect and is indicative of the notion that he was thinking in terms of a concept of mass velocity which, of course, does not exist. He includes the cross-section, $A$, of the column in his definition of mass flow rate, which is a sophomoric error.

We can now articulate the foundation we used for arriving at a value for the column length and, therefore, the column porosity. To do this, we turn to the chromatographic literature where this issue has been thoroughly explored. In [5] herein, we show a crossreference between measured packed column variables and calculated column variables based upon measurements of particle properties. This reference shows, for instance, that particle porosity, $\varepsilon_{p}$, is measured by chromatographers as the ratio, $\varepsilon_{i} /\left(1-\varepsilon_{0}\right)$, where $\varepsilon_{i}$ is the volume fraction of the column occupied by the pores within porous particles and $\left(1-\varepsilon_{0}\right)$ is the volume fraction occupied by the particles. This methodology is in contrast to that typically used by engineers as $\left(1-\rho_{b} / \rho_{s}\right)$ which is based upon the ratio of bulk and solid particle densities. To establish the correct value of the column length, $L$, we adopt an algorithm developed jointly by two renowned chromatographers, Professor Georges Guiochon of the University of Tennessee and distinguished scientist at Argonne National Laboratories, who serendipitously identified the packed column parameter, $P_{t}$, and John Calvin Giddings, former professor of engineering at the University of Utah, whose precise measurements defined an algorithm which pertains to the relationship between $P_{t}$ and $\varepsilon_{t}$ the total column porosity. This is a correlation in the pressure flow relationship when the fluid profile is streamline that accounts for the role of particle porosity and is based upon the independent methodology of measuring fluid linear velocity using injected solutes to determine the rate of change of distance with time, as the fluid passes down the linear distance of the column [5]. This technique does not rely on the value for the diameter of the column which is a critical parameter in determining the fluid velocity when calculated as flow rate divided by column cross-section. Furthermore, to exacerbate the problem of using an imprecise value for column diameter even further in the case of the latter technique, the pressure drop is related to the fourth power in the viscous term and the fifth power in the kinetic term of the characteristic dimension of the fluid channel in Kozeny's theoretical model which was one element of the theoretical model adopted by Ergun and Orning underlying their equation. In our Figure 1(D1), we show a plot of $P_{t}$ versus $\varepsilon_{t}$ for the reported and corrected data for columns A, B, and C, as well as the calibration line for the algorithm. As can be seen from our Table 1 herein, our corrected values for column length, $L$, are as follows: column A, $14.0 \mathrm{~cm}$ to $15.3 \mathrm{~cm} \mathrm{(+9 \% );} \mathrm{column} \mathrm{B,} 23.6 \mathrm{~cm}$ to $25.7 \mathrm{~cm} \mathrm{(+9 \% );} \mathrm{column} \mathrm{C,} 8.5 \mathrm{~cm}$ to $9.4 \mathrm{~cm}(+10 \%)$. These result in increased values of the column porosities as follows: column A, 0.35 to 0.403 ( $+15 \%)$; column $\mathrm{B}, 0.33$ to 0.384 $(+16 \%)$; column C, 0.352 to $0.412(+17 \%)$. As we can see in the plot, the corrected values for column porosity are credible because the corresponding values for the parameter, $P_{t}$, fall in a range greater than 100, approximately, which is typical for these rigid, spherical nonporous particles and, accordingly, fall on the calibration line of the algorithm. The reported values for column porosity, on the other hand, have corresponding values for the parameter, $P_{t}$, which fall in the range below a value of 75 which are too low and, therefore, the corresponding porosity values are not credible. To further justify this correction we point out that, if instead of placing all of the uncertainty in the nominally reported values for the equation variables into just the value of the column length, $L$, alone, we could have divided the total cumulative effect of the tolerances equally over all the nominally reported values, in 
which case the correction would melt into the baseline when the error of the measurement is considered.

\subsection{Ergun's Calibration Columns with Irregularly Shaped} Nonporous Particles. In his 1951 publication, the second in the series of three, Ergun as the sole author on this publication included a host of experimental results all using nitrogen gas as the fluid. His major focus in these experiments was to demonstrate that his newly minted equation could be used to determine the external porosity of porous particles. To validate this, he selected a category of particles which he used to "validate his equation." He termed these particles "Crushed Nonporous Solids" and he proceeded to demonstrate a "comparison of methods" to that of what he called "water displacement." In other words, he crushed porous particles to a particle size that was small enough to render them nonporous, packed these particles into columns, measured their pressure drops under varying flow rates of nitrogen gas, used his equation to back-calculate the external porosity of the column, and, from the weight of the particles used to pack the column, back-calculated the solid particle density of the crushed nonporous particles. He compared this back-calculated result for particle solid density to another independent method which he called "water displacement" and because both techniques returned the same value for the particle solid density, he concluded that this "validated" his equation. In our Figure 2(A1) we show our plots which confirm these results using the version of the equation which uses our values of $d_{p}$ as the characteristic dimension of the particles. In his validation, however, Ergun used the surface area of the particles, $S_{v}$, to represent the solids in the column, thus avoiding the need to deal with the impact of particle morphology in his equation. In fact, he articulated this fact clearly in his 1949 paper when discussing expanded beds. The authors in that paper, Ergun and Orning, point out that "independent knowledge of $S_{v}$ is not necessary. Experimental data for the fixed beds give values of $\alpha S_{v}{ }^{2}$ and $\beta S_{v}$. All dependence on particle size and shape is embedded in these quantities." Accordingly, this validation applies only to his equation in the more basic form in which the particle contribution was represented by $S_{v}$ rather than $d_{p}$. Therefore, his equation, even after this validation exercise, was still uncalibrated with respect to the coefficients of $k_{1}$ and $k_{2}$ which only exist in the form of his equation that contains $d_{p}$ as the characteristic dimension of the solids packed into the column. Because the intercepts of the reported and corrected data sets are identical, our Figure 2(A1), which is based upon the corrected data set, confirms that our corrected values for $k_{1}$ and $k_{2}$ compare favorably to the independent method of water displacement referenced in the Ergun papers.

Ergun did not report either the particle surface area or the average particle size in this 1951 publication. Rather, he specified only the average screen opening in the vibratory screens that he used to classify the particle size fractions of these irregularly sized pulverized particles. Therefore, since we have already determined the calibrated values for the sum of $k_{1}$ and $k_{2}$ from his calibration columns above using spherical particles, we can now use this calibrated value to back-calculate for the actual particle size using a shape factor or sphericity factor, $\Omega_{p}$, for the irregular particles. There were a total of four different particle sizes, the details for which were set forth in his Table 1 of the 1951 paper. Using the same methodology as in our Table 1, we have documented the author's reported data for these columns and used the same correction procedure to enable the identification of the shape factor $\Omega_{p}$. Our Figure 2(A2) shows that the reported data set does not yield consistent values for either of the coefficients $k_{1}$ or $k_{2}$.

Finally, we report the balance of the author's reported data, using the same methodology as above, and display the results in our Figures 3(A1) and 3(A2). This includes the balance of data in the 1951 paper as well as the data presented in the 1952 paper. As can be seen from the plots a comparison between our corrected values and Ergun's reported values shows the arbitrary nature of Ergun's methodology of identifying the value of the residual coefficients $k_{1}$ and $k_{2}$. In our Table 2 we show a summary for all the data including both reported and corrected with a crossreference for our column numbers and the corresponding designation in the Ergun papers.

5.5. The Erroneous Values of 150 and 1.75 Explained. To clearly establish the incorrect foundation underlying the values of 150 for $k_{1}$ and 1.75 for $k_{2}$, announced in the 1952 Ergun papers, we repeat from above the equation for $f_{v}$, the viscous friction factor, which was the equation used in the 1952 paper to generate the values of 150 and 1.75 for the residual coefficients. Ergun called it his "least squares" extrapolation in that paper. Conisder

$$
f_{v}=k_{1}+k_{2} R_{\mathrm{em}} .
$$

Substituting for $f_{v}$ and $R_{\mathrm{em}}$ gives

$$
\frac{\Delta P_{m} \varepsilon_{0}{ }^{3} d_{p}{ }^{2}}{L \mu_{s}{ }^{m}\left(1-\varepsilon_{0}\right)^{2} \eta}=k_{1}+\frac{k_{2} \mu_{s} d_{p} \rho_{f}}{\left(1-\varepsilon_{0}\right) \eta} .
$$

On closer inspection of (34) we can see the problem with Ergun's methodology of extracting the residual coefficients in his equation from his reported data. Note that we have represented the pressure drop with the subscript, $m$, to indicate that it is not the theoretical pressure drop because there is an imbalance in the reported data in the Ergun papers.

(a) Firstly, $\Delta P_{m}$ represents the measured pressure drop when the fluid velocity is $\mu_{s}{ }^{m}$, the superficial velocity at the mean pressure which is separately identified on the left hand side of the equation. But the velocity which Ergun measured was not the velocity at the mean pressure. It was the velocity at STP, represented by the value of $\mu_{s}$ embedded in the value of the modified Reynolds number, on the right hand side of (34). Therefore, there is a mismatch in the equation and it is not balanced with respect to the specific values of velocity on either side of the equalization sign.

(b) Secondly, the left hand side of (34) does not have the fluid density identified as a separate term. The right 


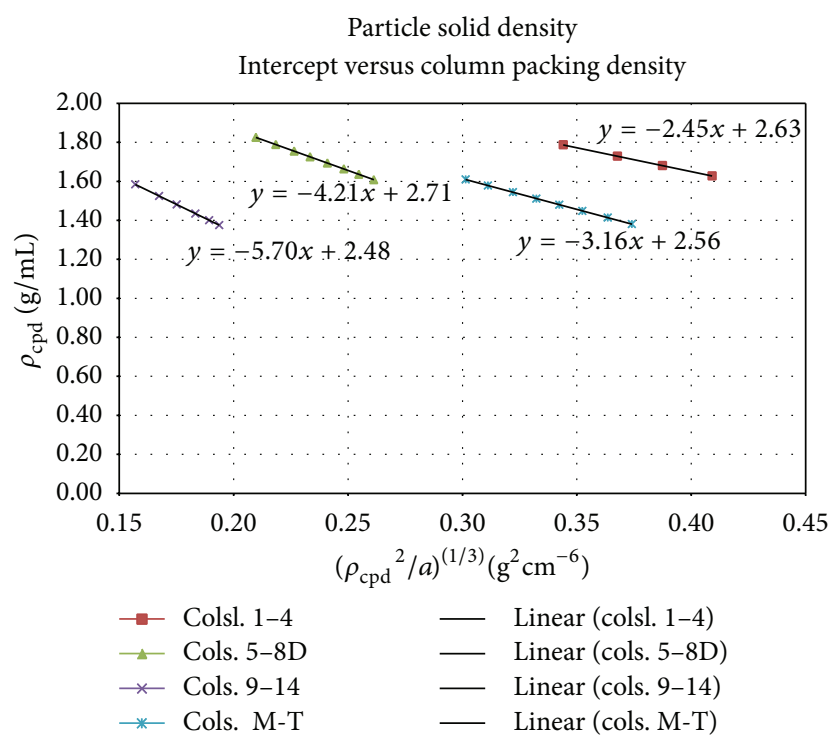

(A1)

Data reexpressed in dimensionless parameters-validation columns Plotted according to Ergun's (13b) in the 1952 paper

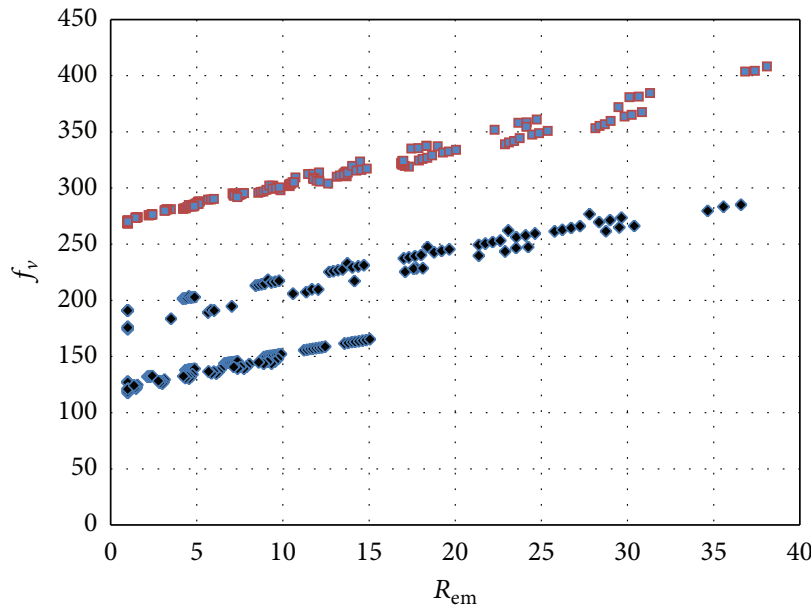

- Reported data

- Corrected data

FIGURE 2

hand side of the equation has the fluid density at STP identified, which is what was measured, but is not representative of the value for this parameter embedded in the measured pressure drop. Accordingly, the measured pressure drop is not normalized for the embedded fluid density at the mean pressure. Therefore, the equation is not balanced with respect to this variable either. This is corroborated further by Ergun's comment expressed in the 1952 paper. "The effect of density is already contained in G."

(c) Thirdly, in the case of the spherical particles in the 1949 columns, the nominal value of the column porosity on both sides of the equation was not sufficiently accurate to produce an accurate backcalculation for the value of $\left(k_{1}+k_{2}\right)$.

(d) Fourthly, in the case of the irregularly shaped particles of the 1951 and 1952 columns, the use of the average screen opening in the place of the value of $d_{p}$, the spherical particle diameter equivalent, on both sides of the equation, leads to erroneous values for the back-calculated value of $\left(k_{1}+k_{2}\right)$.

Accordingly, the intercept and slope of the straight line generated by (34), which is how Ergun derived his values for the coefficients in 1952, are not quantitatively accurate with respect to the combination of viscous and kinetic contributions in the total pressure drop measured, because the 
Data reexpressed in dimensionless parameters-irregularly shaped particles

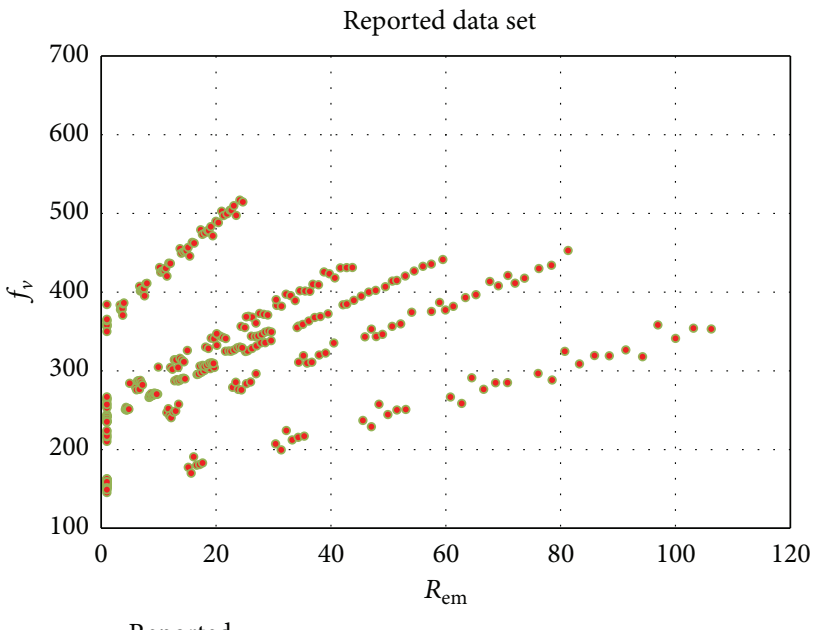

- Reported
Data reexpressed in dimensionless parameters-irregularly shaped particles

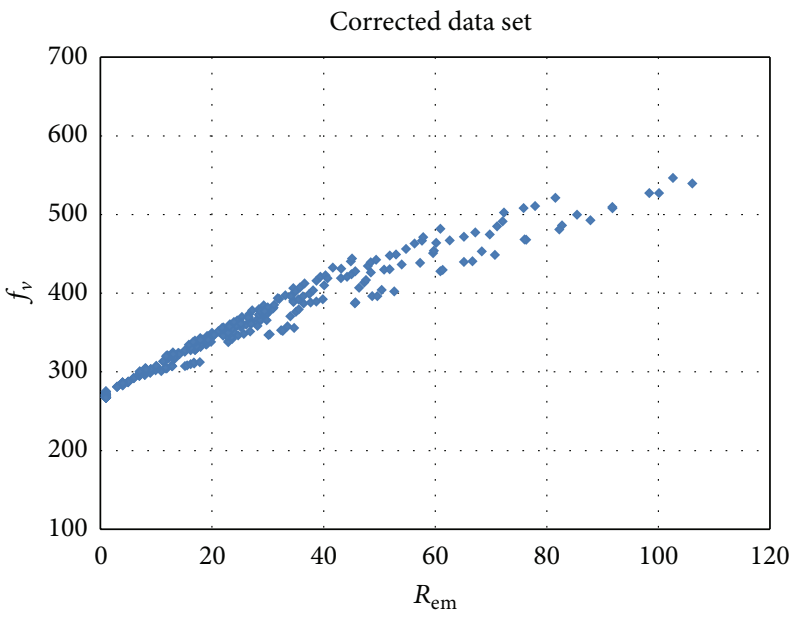

- Corrected equation has not been properly balanced with regard to the specific values of fluid velocity, fluid density, fluid viscosity, column porosity (in the case of the spherical particles), and spherical particle diameter equivalent (in the case of the irregularly shaped particles).

\section{Conclusions}

Of the 640 experiments referred to by Ergun in his 1952 paper which he claims was the foundation for his conclusion that the values of $k_{1}$ and $k_{2}$ were 150 and 1.75 , respectively, we have reviewed approximately $83 \%$ in this review paper. This includes all of his measurements in which nitrogen gas was used as the fluid and represents a total of 78 packed columns and more than 300 individual experimental runs. Our results of extensive analysis and corrections show that the experimental results reported in the Ergun papers, when properly interpreted in view of the experimental protocol used by the authors, generate a value for the sum of $k_{1}$ and $k_{2}$ close to 270 , a value which is, to a first approximation, almost two times greater than that proclaimed by Ergun.

In our extraction procedure for the residual coefficients, $k_{1}$ and $k_{2}$, we use the measured equation of the line reported in the Ergun papers. However, we reject the use of Ergun's mysterious multiplier, $A / \rho_{f}$ stp, which incorrectly treats the fluid density as a constant at all fluid flow rates represented by the fluid density at STP. Instead, we use a constant reference temperature to set the fluid viscosity at a constant which is independent of column pressure and fluid flow rate and, thus, is more representative of what was actually happening within the column when the pressure drop measurements were taken. This allows us to represent the measured data in terms which are not ambiguous with respect to the values of $k_{1}$ and $k_{2}$. Initially, however, we calibrate each column at the boundary condition where both of the value of the modified
Reynolds number and the fluid velocity are unity. This calibration procedure normalizes the equation for all variables and identifies the true value of the sum of the coefficients $k_{1}$ and $k_{2}$. In the case of the 1949 columns, we also correct for the nominal values of the variables reported which are involved in the calculation of the column porosity values, producing the credible range of values for column porosity of 0.38 to 0.41 for the three columns in our study from that paper. We accomplish this by using an independent frame of reference for spherical nonporous particles developed independently by chromatographers which relates pressure gradient and particle porosity to fluid velocity in streamline flow and the accuracy of which is commensurate with the sensitive nature of the pressure flow relationship in the Ergun equation. This establishes the value of the corresponding column porosity in these columns when the pressure measurements were taken. In the case of the other columns reported in the 1951 and 1952 papers which were made from irregularly shaped particles, our back-calculations yielded a range of values of 0.70-1.25 for particle sphericity, $\Omega_{p}$. Because the Ergun papers for these irregularly shaped particles do not provide any independent validation for the spherical particle diameter equivalent, $d_{p}$, other than our back-calculated values based on the reported pressure drop data, we cannot use them to independently assess the values of the residual coefficients in the equation. This is a result of the fact that there are two unknowns and only one equation. The only conclusion that we can express relative to these columns is that the backcalculated sphericity values appear reasonable for pulverized irregularly shaped particles and, accordingly, the empirical data for these columns would appear to support the values for $k_{1}$ and $k_{2}$ identified in the calibration columns of the 1949 paper containing the spherical particles.

The relationship between measured pressure drop and the other equation variables is of such a sensitive nature in 


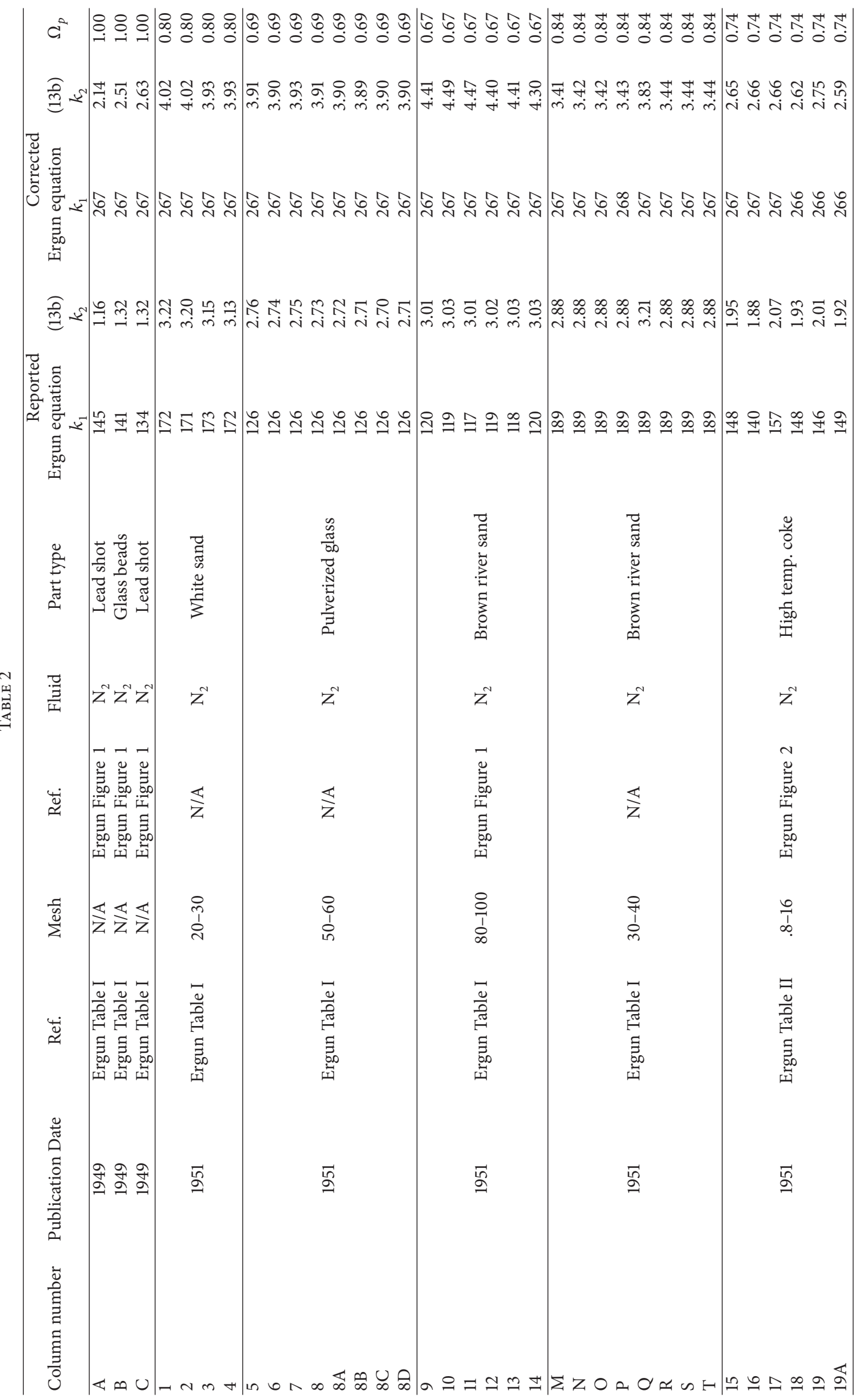




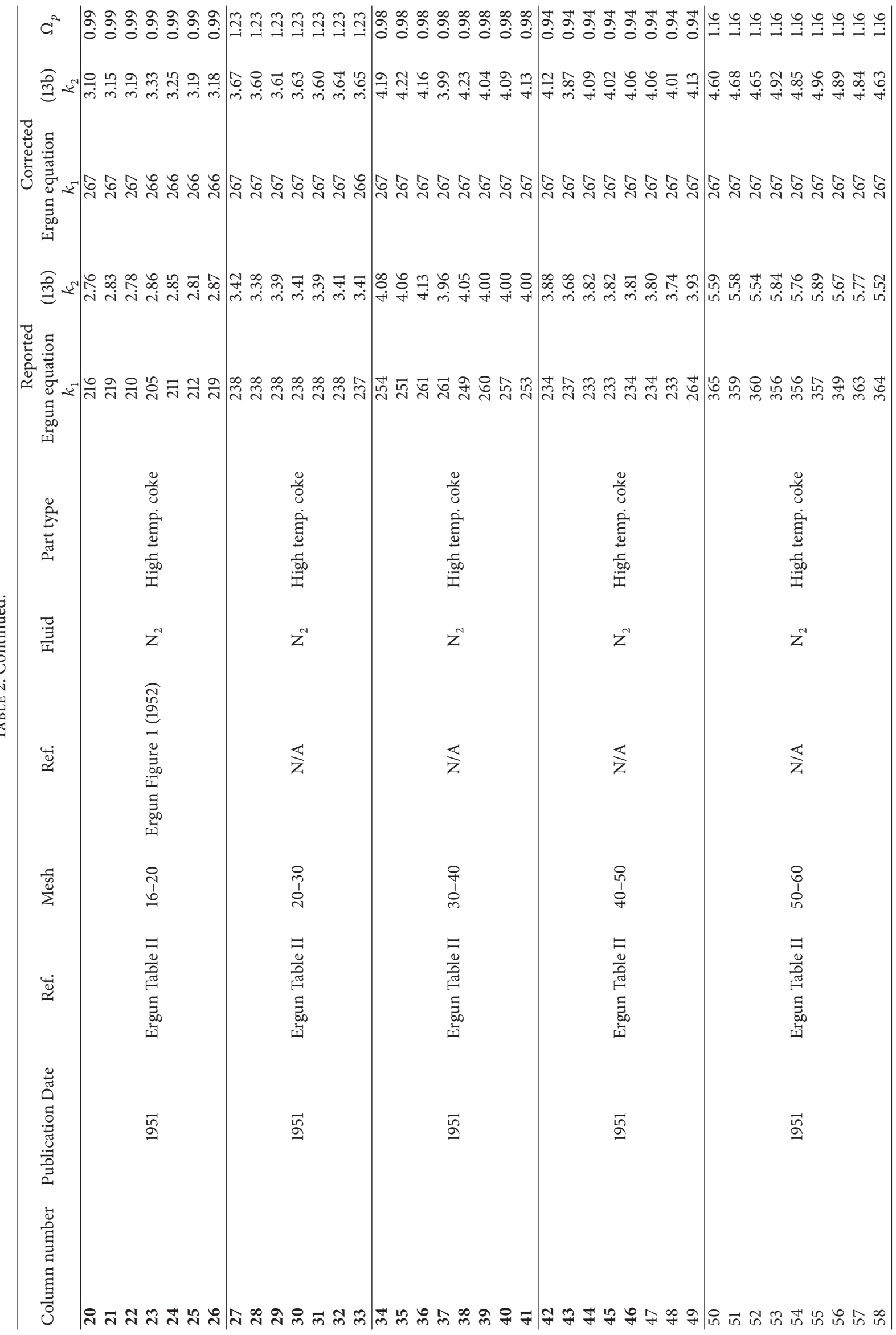




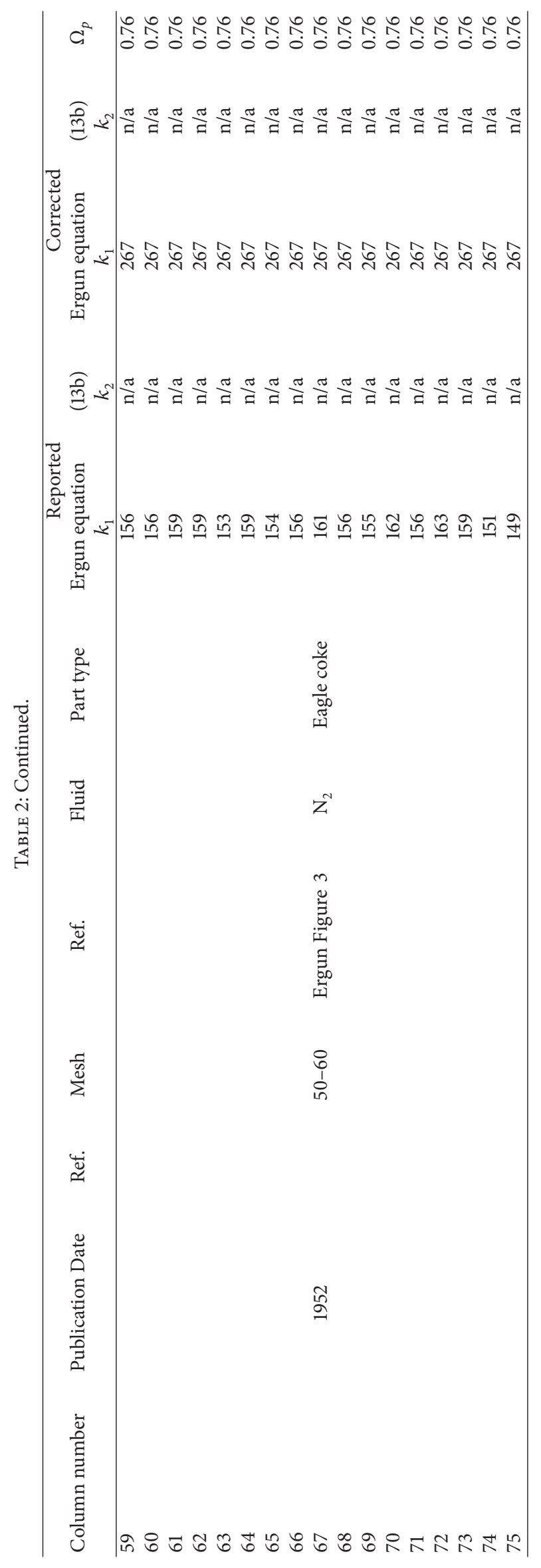


the author's equation that unless one is willing to go into the excruciating details exhibited by our analysis herein, one should not even attempt to express any conclusion as to the value of the residual coefficients. This would have been an admirable, understandable, and correct course of action for Ergun to have taken in 1952 based upon the experimental protocol that he chose and the ambiguous manner with respect to the embedded values of the coefficients $k_{1}$ and $k_{2}$ that he used to characterize his empirical data. On the contrary, however, he did not take this position but rather undertook a course of action which demonstrated an inherent lack of full disclosure. Firstly, his mean pressure postulate embedded as a cornerstone of his equation does not exist anywhere in nature, and, therefore, it cannot be measured. Secondly, he became so obsessed with the mass flow rate of the fluid at the exit of the column, as a means to establish a correlation with fluid density inside the column, that he eventually erroneously comingled it with the cross-section of the column. Thirdly, his experimental protocol, while being readily applicable to incompressible fluids (liquids), does not work when that fluid is a gas, without significant modification. Finally, his use of nominal values for the equation variables responsible for the column porosity calculation in the columns packed with spherical particles and spherical particle diameter equivalent in the case of the irregular particles created a discrepancy in calculation in the reported data set whose cumulative effect not only resulted in values for the residual coefficients that were incorrect, but also resulted in values which were entirely arbitrary.

The errors expressed in the Ergun papers which are, in part, responsible for the discrepancy in the values of the equation coefficients can be catalogued as follows.

(1) In the 1949 paper, the ordinate of the plots displayed in Figures 1, 2, and 3 has the fluid velocity displayed with the symbol $U_{m}$. This is not the velocity at the mean pressure, $u_{m}$, specified in his equation.

(2) In the 1951 paper, the ordinate of the plots displayed in Figures 1 and 2 has the fluid velocity displayed with the symbol $U_{m}$. This is not the velocity at the mean pressure.

(3) In the 1951 paper, the abscissa of the plots displayed in Figures 1 and 2 has the volumetric flow rate displayed, not the mass flow rate as specified in the text.

(4) In the 1952 paper, Figure 4 has the symbol $U_{m}$ displayed on the ordinate of the plot but the text uses the symbol $U$ in (10) to represent the same variable with no attempt by the author to reconcile the two.

(5) In the glossary of terms at the end of the 1952 paper, the author defines the mass flow rate $G=\rho U$ where $U$ represents the superficial velocity of the fluid. This is an incorrect definition for mass flow rate which does not involve the column cross-section embedded in the formula for fluid velocity. Thus the author confuses fluid flow rate and fluid velocity.

(6) In the glossary of terms at the end of the 1952 paper, the author defines the meaning of the symbol
$U_{m}$ as "superficial fluid velocity measured at average pressure." Nowhere in any of the three papers do the authors identify this measured value or how they carried out the measurement.

(7) In the 1949 paper, the authors used nominal values for column length and particle solid density which rendered a nominal column porosity value not suitable for a quantitative determination of the residual equation coefficients.

(8) In the 1951 and 1952 papers, the author did not disclose his values for the particle sphericity or, alternatively, his assumed value for the average spherical particle diameter equivalent. Instead, he used the average screen opening of the vibratory screens used to classify the particle fractions. This technique does not facilitate a quantitative determination of the equation residual coefficients.

(9) The authors in all of the Ergun papers reported their measured values ambiguously with respect to the embedded values of $k_{1}$ and $k_{2}$. They accomplished this by reporting ratios in their straight line plots of the empirical data rather than the actual values for each variable obtained in the measurements. These straight line plots yield values for $a$ and $b$, the intercept and slope, respectively, which do not provide unique values for the coefficients $k_{1}$ and $k_{2}$, when all the details of their embedded variables are not specifically identified.

(10) Ergun made no serious attempt in his 1952 paper to identify experimentally the value of either of the coefficients individually. In order to do this he would have had to have taken pressure drop measurements at either end of the modified Reynolds number spectrum where the measured pressure drop would be dominated by the contribution of either viscous or kinetic sources, something he did not do. On the contrary, he took all his measurements in the transition region of the fluid flow regime where the pressure drop measurements contained significant contributions from both viscous and kinetic sources.

(11) Finally, Ergun and Orning got the wrong value for $k_{1}$ because they used nominal values to calculate the column porosity in the 1949 columns and because Ergun used the screen opening dimensions instead of the spherical particle diameter equivalent in the columns packed with irregularly shaped particles. In addition, Ergun got the wrong value for $k_{2}$ because he assumed a constant fluid density at every flow rate in a given column. But aside from this discrepancy concerning the value of $k_{2}$, the empirical data reported in the Ergun papers when properly evaluated demonstrates that the Ergun equation is not valid with respect to the kinetic side of the equation because, contrary to Ergun's proclamation in the 1952 paper, the value of $k_{2}$ is not constant.

If the errors involved in the methodology and science were the only issues surrounding the Ergun papers, other scientists 
might have figured them out before now, some sixty years down the road. The disingenuous manner in which the papers were written, on the other hand, has probably more to do with the confusion that exists today concerning the values of the coefficients in the Ergun equation than any other single parameter. This included Ergun's continued attempts in all three papers to create the illusion that he was measuring the velocity of the fluid at the mean pressure, $U_{m}$, a symbol he eventually defined in his final paper in 1952 but was by then a blatant misrepresentation of what he was actually measuring, thus playing hide and go seek with his mean pressure postulate which he never dealt with in a straightforward manner in any of the three papers. His omission of details surrounding the way in which he deployed his two capillary flow-meters did little to inform the reader on what his the real experimental protocol represented with respect to measured fluid velocity. And, finally, most egregious of all, the lack of transparency surrounding the use of his mysterious multiplier $(5,790)$ was, up until now, a real barrier to understanding the true meaning of any of the data reported in his papers.

Tragically, the most damaging legacy over the past 60 years, approximately, of Ergun's erroneous values for the constants in the equation, however, is that the scientific literature in this arena has become filled with the contribution of authors who have claimed that their work has corroborated these values and, in addition, has been the starting point for many derivations which purport to represent legitimate advances in the understanding of fluid mechanics in porous media. This reality confirms that, unfortunately, once something gets into print, whether fact or fiction, it takes on a life of its own.

\section{Conflict of Interests}

The author declares that there is no conflict of interests regarding the publication of this paper.

\section{Acknowledgment}

Sincere gratitude is expressed to Tony Edge, senior scientist at ThermoFisher Scientific, for his critique of the paper and helpful discussions on many of the important topics herein.

\section{References}

[1] H. Darcy, Les Fontaines Publiques de la Ville de Dijon, Victor Dalmont, Paris, France, 1856.

[2] J. Kozeny, "Uber kapillare Leitung des wassers in Böden," Sitzungsberichte der Kaiserlichen Akademie der Wissenschaften, vol. 136, pp. 271-306, 1927.

[3] F. E. Blake, "The resistance of packing to fluid flow," Transaction of American Institute of Chemical Engineers, vol. 14, pp. 415-421, 1922.

[4] H. M. Quinn, "Reconciliation of packed column permeability data-Part 1. the teaching of Giddings revisited," Special Topics and Reviews in Porous Media, vol. 1, no. 1, pp. 79-86, 2010.
[5] H. M. Quinn, "Reconciliation of packed column permeability data, column permeability as a function of particle porosity," Journal of Materials, vol. 2014, Article ID 636507, 22 pages, 2014.

[6] P. C. Carman, "Flow through granular beds," Transactions of the Institution of Chemical Engineers, vol. 15, pp. 150-156, 1973.

[7] J. C. Giddings, Dynamics of Chromatography, Part I. Principles and Theory, Marcel Dekker, New York, NY, USA, 1965.

[8] J. C. Giddings, Unified Separation Science, Wiley, New York, NY, USA, 1991.

[9] G. Guiochon, S. G. Shirazi, and A. M. Katti, Fundamentals of Preparative and Nonlinear Chromatography, Academic Press, Boston, Mass, USA, 2nd edition, 2006.

[10] A. E. Scheidegger, The Physics of Flow Through Porous Media, MacMillan Company, New York, NY, USA, 1957.

[11] R. B. Bird, W. E. Stewart, and E. N. Lightfoot, Transport Phenomena, John Wiley \& Sons, New York, NY, USA, 1960.

[12] J. Happel and H. Brenner, Low Reynolds Number Hydrodynamics, Prentice-Hall, 1965.

[13] S. W. Churchill, Viscous Flows: The Practical Use of Theory, Butterworks, 1988.

[14] U. D. Neue, HPLC Columns, Theory, Technology and Practice, Wiley-VCH, New York, NY, USA, 1997.

[15] F. A. L. Dullien, Porous Media, Fluid Transport and Pore Structure, Acedemic Press, 2nd edition, 1979.

[16] M. Rhodes, Introduction to Particle Technology, John Wiley \& Sons, 1998.

[17] S. Ergun and A. A. Orning, "Fluid flow through randomly packed columns and fluidized beds," Industrial \& Engineering Chemistry, vol. 4, no. 6, pp. 1179-1184, 1949.

[18] S. Ergun, "Determination of particle density of crushed porous solids," Analytical Chemistry, vol. 23, no. 1, pp. 151-156, 1951.

[19] S. Ergun, "Fluid flow through packed column," Chemical Engineering Progress, vol. 49, pp. 89-94, 1952.

[20] D. I. Hitchcock, Journal of General Physiology, vol. 9, p. 735, 1926.

[21] M. Leva and M. Grummer, "Pressure drop through packed tubes, part I, a general correlation,” vol. 43, pp. 549-554, 1947.

[22] S. P. Burke and W. B. Plummer, "Gas flow through packed columns," Industrial and Engineering Chemistry, vol. 20, pp. $1196-1200,1923$.

[23] A. O. Oman and K. M. Watson, "Pressure drops in granular beds," National Petroleum News, vol. 36, pp. R795-R802, 1944. 

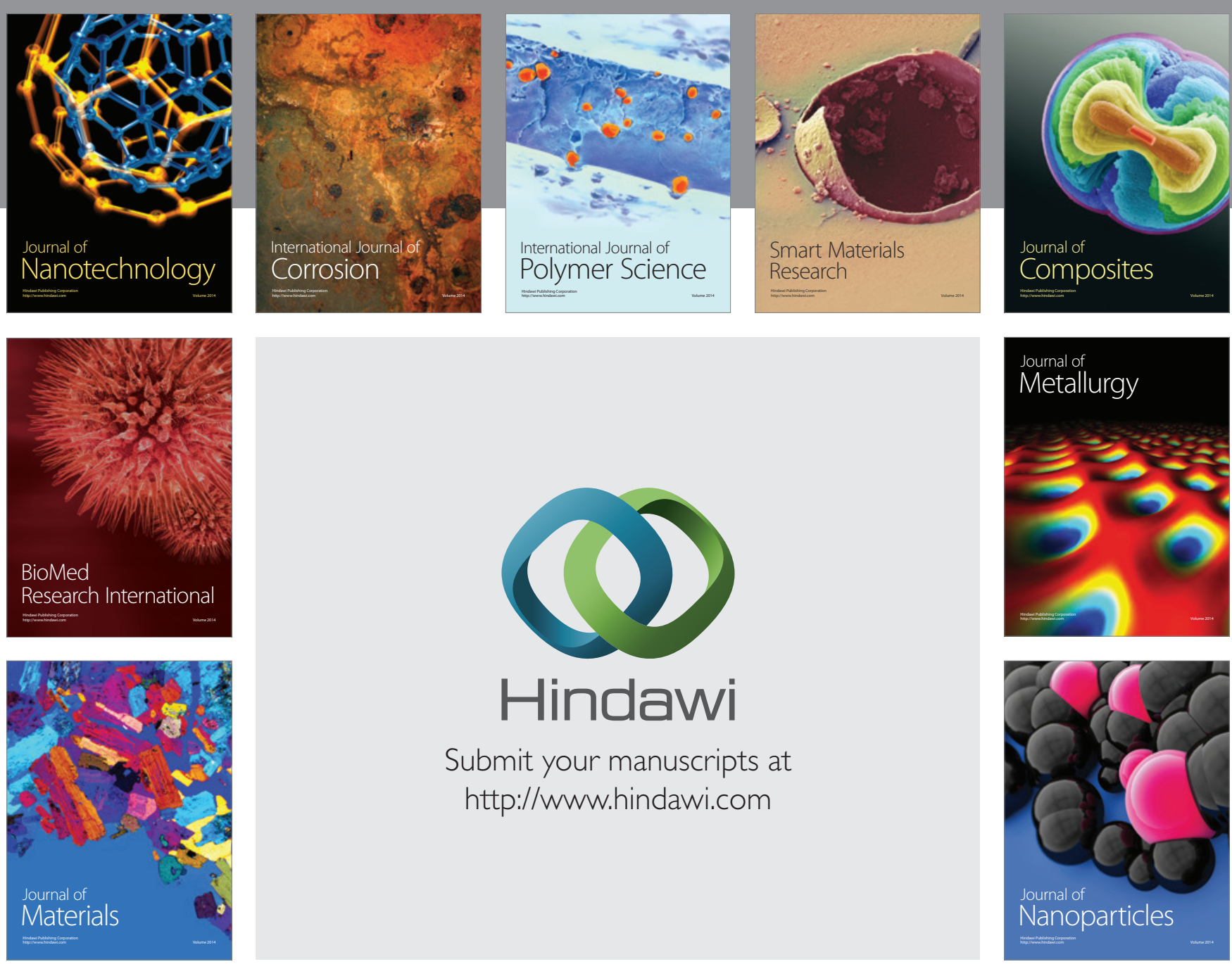

Submit your manuscripts at http://www.hindawi.com
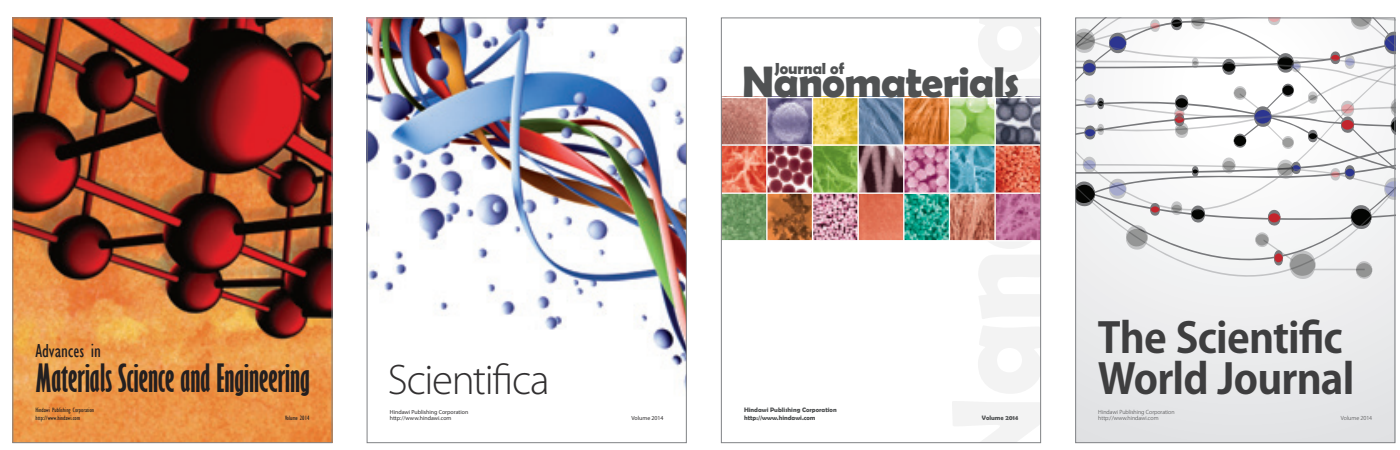

\section{The Scientific World Journal}
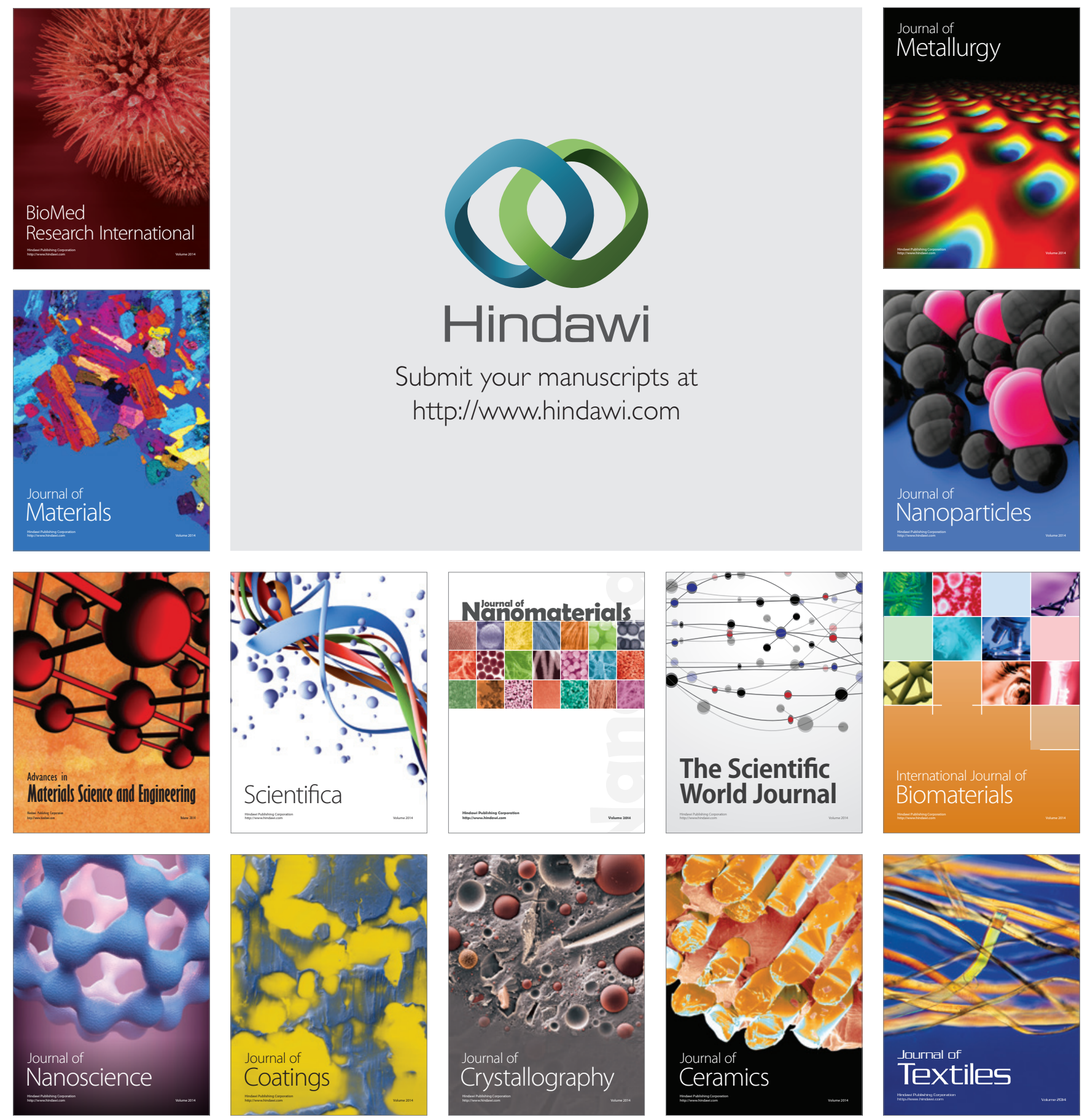